BULLETIN (New Series) OF THE

AMERICAN MATHEMATICAL SOCIETY

Volume 48, Number 1, January 2011, Pages 53-84

S 0273-0979(2010)01321-5

Article electronically published on October 4, 2010

\title{
MULTIVARIATE STABLE POLYNOMIALS: THEORY AND APPLICATIONS
}

\author{
DAVID G. WAGNER
}

In memoriam of Julius Borcea

\begin{abstract}
Univariate polynomials with only real roots, while special, do occur often enough that their properties can lead to interesting conclusions in diverse areas. Due mainly to the recent work of two young mathematicians, Julius Borcea and Petter Brändén, a very successful multivariate generalization of this method has been developed. The first part of this paper surveys some of the main results of this theory of multivariate stable polynomials - the most central of these results is the characterization of linear transformations preserving stability of polynomials. The second part presents various applications of this theory in complex analysis, matrix theory, probability and statistical mechanics, and combinatorics.
\end{abstract}

\section{INTRODUCTION}

The editor of the Bulletin of the American Mathematical Society has asked me to survey the recent work of Julius Borcea and Petter Brändén on their multivariate generalization of the theory of univariate polynomials with only real roots, and its applications. It is exciting work - elementary but subtle, and with spectacular consequences. Borcea and Brändén take center stage but there are many other actors, many of whom I am unable to mention in this brief treatment. Notably, Leonid Gurvits provides a transparent proof of a vast generalization of the famous van der Waerden Conjecture.

Space is limited and I have been advised to use "Bourbaki style", and so this is an account of the essentials of the theory and a few of its applications, with complete proofs as far as possible. Some relatively straightforward arguments have been left as exercises to engage the reader, and some more specialized topics are merely sketched or even omitted. For the full story and the history and context of the subject one must go to the references cited, the references they cite, and so on. The introduction of [4, in particular, gives a good account of the genesis of the theory.

Received by the editors May 17, 2010, and, in revised form, June 12, 2010.

2010 Mathematics Subject Classification. Primary 32A60; Secondary 05A20, 05B35, 15A45, 15A48, 60G55, 60K35.

Research supported by NSERC Discovery Grant OGP0105392.

(C)2010 American Mathematical Society Reverts to public domain 28 years from publication 
Here is a brief summary of the contents. Section 2 introduces stable polynomials, gives some examples, presents their elementary properties, and develops multivariate generalizations of two classical univariate results: the Hermite-KakeyaObreschkoff and Hermite-Biehler Theorems. We also state the Pólya-Schur Theorem characterizing multiplier sequences, as this provides an inspiration for much of the multivariate theory. Section 3 restricts attention to multiaffine stable polynomials: we present a characterization of multiaffine real stable polynomials by means of parameterized quadratic inequalities, and characterize those linear transformations which take multiaffine stable polynomials to stable polynomials. In Section 4 we use parts of the forgoing for Borcea and Brändén's splendid proof of the Grace-WalshSzegö Coincidence Theorem. In Section 5, the Grace-Walsh-Szegö Theorem is used to extend the results of Section 3 from multiaffine to arbitrary stable polynomials. This culminates in an amazing multivariate generalization of the Pólya-Schur Theorem, the proof of which requires the development of a multivariate extension of the Szász Principle. We then turn to applications of the theory. Section 6 presents Borcea and Brändén's resolution of some matrix-theoretic conjectures of Johnson. Section 7 presents the derivation by Borcea, Brändén, and Liggett of negative association inequalities for the symmetric exclusion process, a fundamental model in probability and statistical mechanics. Section 8 presents Gurvits's sweeping generalization of the van der Waerden Conjecture. Finally, Section 9 briefly mentions a few further topics that could not be included fully for lack of space.

I thank Petter Brändén kindly for his helpful comments on preliminary drafts of this paper.

\section{Stable polynomials}

We use the following shorthand notation for multivariate polynomials. Let $[m]=$ $\{1,2, \ldots, m\}$, let $\mathbf{x}=\left(x_{1}, \ldots, x_{m}\right)$ be a sequence of indeterminates, and let $\mathbb{C}[\mathbf{x}]$ be the ring of complex polynomials in the indeterminates $\mathbf{x}$. For a function $\alpha$ : $[m] \rightarrow \mathbb{N}$, let $\mathbf{x}^{\alpha}=x_{1}^{\alpha(1)} \cdots x_{m}^{\alpha(m)}$ be the corresponding monomial. For $S \subseteq[m]$ we also let $\mathbf{x}^{S}=\prod_{i \in S} x_{i}$. Similarly, for $i \in[m]$, let $\partial_{i}=\partial / \partial x_{i}$, let $\boldsymbol{\partial}=\left(\partial_{1}, \ldots, \partial_{m}\right)$, let $\boldsymbol{\partial}^{\alpha}=\partial_{1}^{\alpha(1)} \cdots \partial_{m}^{\alpha(m)}$, and let $\boldsymbol{\partial}^{S}=\prod_{i \in S} \partial_{i}$. The $\mathbf{x}$ indeterminates are always indexed by $[m]$. The set $\mathbb{N}^{m}$ is equipped with its coordinatewise partial order. The constant functions on $[m]$ with images 0 or 1 are denoted by $\mathbf{0}$ and $\mathbf{1}$, respectively. For each $i \in[m]$, let $\delta_{i} \in \mathbb{N}^{m}$ have $\delta_{i}(i)=1$ and $\delta_{i}(j)=0$ if $j \neq i$. For $\alpha \in \mathbb{N}^{m}$, let $|\alpha|=\sum_{i=1}^{m} \alpha(i)$. For $\alpha, \beta \in \mathbb{Z}^{m}$, let $\alpha^{\beta}=\prod_{i=1}^{m} \alpha(i)^{\beta(i)}$, when defined (note that $\left.0^{0}=1\right)$. Also, let $\alpha !=\prod_{i=1}^{m} \alpha(i)$ !, and define $(\beta)_{\alpha}=\beta ! /(\beta-\alpha) !$ if $\alpha \leq \beta$ in $\mathbb{N}^{m}$, and $(\beta)_{\alpha}=0$ if $\alpha \not \leq \beta$.

Let $\mathcal{H}=\{z \in \mathbb{C}: \operatorname{Im}(z)>0\}$ denote the open upper half of the complex plane, and let $\overline{\mathcal{H}}$ be the closure of $\mathcal{H}$ in $\mathbb{C}$. A polynomial $f \in \mathbb{C}[\mathbf{x}]$ is stable provided that either $f \equiv 0$ identically, or whenever $\mathbf{z}=\left(z_{1}, \ldots, z_{m}\right) \in \mathcal{H}^{m}$, then $f(\mathbf{z}) \neq 0$. We use $\mathfrak{S}[\mathbf{x}]$ to denote the set of stable polynomials in $\mathbb{C}[\mathbf{x}]$, and $\mathfrak{S}_{\mathbb{R}}[\mathbf{x}]=\mathfrak{S}[\mathbf{x}] \cap \mathbb{R}[\mathbf{x}]$ for the set of real stable polynomials in $\mathbb{R}[\mathbf{x}]$. (Borcea and Brändén do not consider the zero polynomial to be stable, but I find the above convention more convenient.)

We rely on the following essential fact at several points.

Hurwitz's Theorem (Theorem 1.3.8 of [16]). Let $\Omega \subseteq \mathbb{C}^{m}$ be a connected open set, and let $\left(f_{n}: n \in \mathbb{N}\right)$ be a sequence of functions, each analytic and nonvanishing on $\Omega$, which converges to a limit $f$ uniformly on compact subsets of $\Omega$. Then $f$ is either nonvanishing on $\Omega$ or identically zero. 
Consequently, a polynomial obtained as the limit of a convergent sequence of stable polynomials is itself stable.

\subsection{Examples.}

Proposition 2.1 (Proposition 2.4 of [1]). For $i \in[m]$, let $A_{i}$ be an $n$-by-n matrix, let $x_{i}$ be an indeterminate, and let $B$ be an $n$-by-n matrix. If $A_{i}$ is positive semidefinite for all $i \in[m]$ and $B$ is Hermitian, then

$$
f(\mathbf{x})=\operatorname{det}\left(x_{1} A_{1}+x_{2} A_{2}+\cdots+x_{m} A_{m}+B\right)
$$

is real stable.

Proof. Let $\bar{f}$ denote the coefficientwise complex conjugate of $f$. Since $\overline{A_{i}}=A_{i}^{\top}$ for all $i \in[m]$, and $\bar{B}=B^{\top}$, it follows that $\bar{f}=f$, so that $f \in \mathbb{R}[\mathbf{x}]$. By Hurwitz's Theorem and a routine perturbation argument, it suffices to prove that $f$ is stable when each $A_{i}$ is positive definite. Consider any $\mathbf{z}=\mathbf{a}+\mathbf{i b} \in \mathcal{H}^{m}$, with $\mathbf{a}, \mathbf{b} \in \mathbb{R}^{m}$ and $b_{i}>0$ for all $i \in[m]$ (abbreviated to $\mathbf{b}>\mathbf{0}$ ). Now $Q=\sum_{i=1}^{m} b_{i} A_{i}$ is positive definite, and hence has a positive definite squareroot $Q^{1 / 2}$. Also note that $H=$ $\sum_{i=1}^{m} a_{i} A_{i}+B$ is Hermitian, and that

$$
f(\mathbf{z})=\operatorname{det}(Q) \operatorname{det}\left(\mathrm{i} I+Q^{-1 / 2} H Q^{-1 / 2}\right) .
$$

Since $\operatorname{det}(Q) \neq 0$, if $f(\mathbf{z})=0$, then $-\mathrm{i}$ is an eigenvalue of $Q^{-1 / 2} H Q^{-1 / 2}$, contradicting the fact that this matrix is Hermitian. Thus, $f(\mathbf{z}) \neq 0$ for all $\mathbf{z} \in \mathcal{H}^{m}$. That is, $f$ is stable.

Corollary 2.2. Let $Q$ be an $n$-by-m complex matrix, and let $X=\operatorname{diag}\left(x_{1}, \ldots, x_{m}\right)$ be a diagonal matrix of indeterminates. Then $f(\mathbf{x})=\operatorname{det}\left(Q X Q^{\dagger}\right)$ is real stable.

Proof. Let $Q=\left(q_{i j}\right)$, and for $j \in[m]$ let $A_{j}$ denote the $n$-by-n matrix with $h i$ th entry $q_{h j} \bar{q}_{i j}$. That is, $A_{j}=Q_{j} Q_{j}^{\dagger}$ in which $Q_{j}$ denotes the $j$ th column of $Q$. Since each $A_{j}$ is positive semidefinite and $Q X Q^{\dagger}=x_{1} A_{1}+\cdots+x_{m} A_{m}$, the conclusion follows directly from Proposition 2.1.

2.2. Elementary properties. The following simple observation often allows multivariate problems to be reduced to univariate ones, as will be seen.

Lemma 2.3. A polynomial $f \in \mathbb{C}[\mathbf{x}]$ is stable if and only if for all $\mathbf{a}, \mathbf{b} \in \mathbb{R}^{m}$ with $\mathbf{b}>\mathbf{0}, f(\mathbf{a}+\mathbf{b} t)$ is stable in $\mathfrak{S}[t]$.

Proof. Since $\mathcal{H}^{m}=\left\{\mathbf{a}+\mathbf{b} t: \mathbf{a}, \mathbf{b} \in \mathbb{R}^{m}, \mathbf{b}>\mathbf{0}\right.$, and $\left.t \in \mathcal{H}\right\}$, the result follows.

For those who know, Lemma 2.3 states that a polynomial is stable if and only if it is Gårding hyperbolic with hyperbolicity cone containing the positive orthant; see [11, 13].

For $f \in \mathbb{C}[\mathbf{x}]$ and $i \in[m]$, let $\operatorname{deg}_{i}(f)$ denote the degree of $x_{i}$ in $f$.

Lemma 2.4. These operations preserve stability of polynomials in $\mathbb{C}[\mathbf{x}]$.

(a) Permutation: for any permutation $\sigma:[m] \rightarrow[m], f \mapsto f\left(x_{\sigma(1)}, \ldots, x_{\sigma(m)}\right)$.

(b) Scaling: for $c \in \mathbb{C}$ and $\mathbf{a} \in \mathbb{R}^{m}$ with $\mathbf{a}>\mathbf{0}, f \mapsto c f\left(a_{1} x_{1}, \ldots, a_{m} x_{m}\right)$.

(c) Diagonalization: for $\{i, j\} \subseteq[m],\left.f \mapsto f(\mathbf{x})\right|_{x_{i}=x_{j}}$.

(d) Specialization: for $a \in \overline{\mathcal{H}}, f \mapsto f\left(a, x_{2}, \ldots, x_{m}\right)$.

(e) Inversion: if $\operatorname{deg}_{1}(f)=d, f \mapsto x_{1}^{d} f\left(-x_{1}^{-1}, x_{2}, \ldots, x_{m}\right)$.

(f) Differentiation (or Contraction): $f \mapsto \partial_{1} f(\mathbf{x})$. 
Proof. Parts (a), (b), and (c) are clear. Part (d) is also clear in the case that $\operatorname{Im}(a)>0$. For $a \in \mathbb{R}$ apply part (d) with values in the sequence $\left(a+\mathrm{i} 2^{-n}: n \in\right.$ $\mathbb{N}$ ), and then apply Hurwitz's Theorem to the limit as $n \rightarrow \infty$. Part (e) follows from the fact that $\mathcal{H}$ is invariant under the operation $z \mapsto-z^{-1}$. For part (f), let $d=\operatorname{deg}_{1}(f)$, and consider the sequence $f_{n}=n^{-d} f\left(n x_{1}, x_{2}, \ldots, x_{m}\right)$ for all $n \geq 1$. Each $f_{n}$ is stable and the sequence converges to a polynomial, so the limit is stable. Since $\operatorname{deg}_{1}(f)=d$, this limit is not identically zero. This implies that for all $z_{2}, \ldots, z_{m} \in \mathcal{H}$, the polynomial $g(x)=f\left(x, z_{2}, \ldots, z_{m}\right) \in \mathbb{C}[x]$ has degree $d$. Clearly $g^{\prime}(x)=\partial_{1} f\left(x, z_{2}, \ldots, z_{m}\right)$. Let $\xi_{1}, \ldots, \xi_{d}$ be the roots of $g(x)$, so that $g(x)=c \prod_{h=1}^{d}\left(x-\xi_{h}\right)$ for some $c \in \mathbb{C}$. Since $f$ is stable, $\operatorname{Im}\left(\xi_{h}\right) \leq 0$ for all $h \in[d]$. Now

$$
\frac{g^{\prime}(x)}{g(x)}=\frac{d}{d x} \log g(x)=\sum_{h=1}^{d} \frac{1}{x-\xi_{h}} .
$$

If $\operatorname{Im}(z)>0$, then $\operatorname{Im}\left(1 /\left(z-\xi_{h}\right)\right)<0$ for all $h \in[d]$, so that $g^{\prime}(z) \neq 0$. Thus, if $\mathbf{z} \in \mathcal{H}^{m}$, then $\partial_{1} f(\mathbf{z}) \neq 0$. That is, $\partial_{1} f$ is stable.

Of course, by permutation, parts (d), (e), and (f) of Lemma 2.4 apply for any index $i \in[m]$ as well (not just $i=1$ ). Part (f) is essentially the Gauss-Lucas Theorem: the roots of $g^{\prime}(x)$ lie in the convex hull of the roots of $g(x)$.

2.3. Univariate stable polynomials. A nonzero univariate polynomial is real stable if and only if it has only real roots. Let $f$ and $g$ be two such polynomials, let $\xi_{1} \leq \xi_{2} \leq \cdots \leq \xi_{k}$ be the roots of $f$, and let $\theta_{1} \leq \theta_{2} \leq \cdots \leq \theta_{\ell}$ be the roots of $g$. These roots are interlaced if they are ordered so that $\xi_{1} \leq \theta_{1} \leq \xi_{2} \leq \theta_{2} \leq \cdots$ or $\theta_{1} \leq \xi_{1} \leq \theta_{2} \leq \xi_{2} \leq \cdots$. For each $i \in[\ell]$, let $\widehat{g}_{i}=g /\left(x-\theta_{i}\right)$. If $\operatorname{deg}(f) \leq \operatorname{deg}(g)$ and the roots of $g$ are simple, then there is a unique $\left(a, b_{1}, \ldots, b_{\ell}\right) \in \mathbb{R}^{\ell+1}$ such that

$$
f=a g+b_{1} \widehat{g}_{1}+\cdots+b_{\ell} \widehat{g}_{\ell} .
$$

Exercise 2.5. Let $f, g \in \mathfrak{S}_{\mathbb{R}}[x]$ be nonzero and such that $f g$ has only simple roots, let $\operatorname{deg}(f) \leq \operatorname{deg}(g)$, and let $\theta_{1}<\cdots<\theta_{\ell}$ be the roots of $g$. The following are equivalent:

(a) The roots of $f$ and $g$ are interlaced.

(b) The sequence $f\left(\theta_{1}\right), f\left(\theta_{2}\right), \ldots, f\left(\theta_{\ell}\right)$ alternates in sign (strictly).

(c) In $f=a g+\sum_{i=1}^{\ell} b_{i} \widehat{g}_{i}$, all of $b_{1}, \ldots, b_{\ell}$ have the same sign (and are nonzero).

The Wronskian of $f, g \in \mathbb{C}[x]$ is $\mathrm{W}[f, g]=f^{\prime} \cdot g-f \cdot g^{\prime}$. If $f=a g+\sum_{i=1}^{\ell} b_{i} \widehat{g}_{i}$ as in Exercise 2.5, then

$$
\frac{\mathrm{W}[f, g]}{g^{2}}=\frac{d}{d x}\left(\frac{f}{g}\right)=\sum_{i=1}^{\ell} \frac{-b_{i}}{\left(x-\theta_{i}\right)^{2}} .
$$

It follows that if $f$ and $g$ are as in Exercise 2.5(a), then $\mathrm{W}[f, g]$ is either positive for all real $x$, or negative for all real $x$. Since $\mathrm{W}[g, f]=-\mathrm{W}[f, g]$, the condition that $\operatorname{deg}(f) \leq \operatorname{deg}(g)$ is immaterial. Any pair $f, g$ with interlacing roots can be approximated arbitrarily closely by such a pair with all roots of $f g$ simple. It follows that for any pair $f, g$ with interlacing roots, the Wronskian $\mathrm{W}[f, g]$ is either nonnegative on all of $\mathbb{R}$ or nonpositive on all of $\mathbb{R}$.

Nonzero univariate polynomials $f, g \in \mathfrak{S}_{\mathbb{R}}[x]$ are in proper position, denoted by $f \ll g$, if $\mathrm{W}[f, g] \leq 0$ on all of $\mathbb{R}$. For convenience we also let $0 \ll f$ and $f \ll 0$ for any $f \in \mathfrak{S}_{\mathbb{R}}[x]$; in particular $0 \ll 0$. 
Exercise 2.6. Let $f, g \in \mathfrak{S}_{\mathbb{R}}[x]$ be real stable. Then $f \ll g$ and $g \ll f$ if and only if $c f=d g$ for some $c, d \in \mathbb{R}$ not both zero.

Hermite-Kakeya-Obreschkoff (HKO) Theorem (Theorem 6.3 .8 of [16]). Let $f, g \in \mathbb{R}[x]$. Then af $+b g \in \mathfrak{S}_{\mathbb{R}}[x]$ for all $a, b \in \mathbb{R}$ if and only if $f, g \in \mathfrak{S}_{\mathbb{R}}[x]$ and either $f \ll g$ or $g \ll f$.

Hermite-Biehler (HB) Theorem (Theorem 6.3.4 of [16]). Let $f, g \in \mathbb{R}[x]$. Then $g+$ if $\in \mathfrak{S}[x]$ if and only if $f, g \in \mathfrak{S}_{\mathbb{R}}[x]$ and $f \ll g$.

Proofs of $H K O$ and $H B$. It suffices to prove these when $f g$ has only simple roots.

For HKO we can assume that $\operatorname{deg}(f) \leq \operatorname{deg}(g)$. Exercise 2.5 shows that if the roots of $f$ and $g$ are interlaced, then for all $a, b \in \mathbb{R}$, the roots of $g$ and $a f+b g$ are interlaced so that $a f+b g$ is real stable. The converse is trivial if $c f=d g$ for some $c, d \in \mathbb{R}$ not both zero, so assume otherwise. From the hypothesis, both $f$ and $g$ are real stable. If there are $z_{0}, z_{1} \in \mathcal{H}$ for which $\operatorname{Im}\left(f\left(z_{0}\right) / g\left(z_{0}\right)\right)<0$ and $\operatorname{Im}\left(f\left(z_{1}\right) / g\left(z_{1}\right)\right)>0$, then for some $\lambda \in[0,1]$ the number $z_{\lambda}=(1-\lambda) z_{0}+\lambda z_{1}$ is such that $\operatorname{Im}\left(f\left(z_{\lambda}\right) / g\left(z_{\lambda}\right)\right)=0$. Thus $f\left(z_{\lambda}\right)-a g\left(z_{\lambda}\right)=0$ for some real number $a \in \mathbb{R}$. Since $f-a g$ is stable (by hypothesis) and $z_{\lambda} \in \mathcal{H}$, this implies that $f-a g \equiv 0$, a contradiction. Thus $\operatorname{Im}(f(z) / g(z))$ does not change sign for $z \in \mathcal{H}$. This implies Exercise 2.5(c): all the $b_{i}$ have the same sign (consider $f / g$ at the points $\theta_{i}+\mathrm{i} \epsilon$ for $\epsilon>0$ approaching 0 ). Thus, the roots of $f$ and $g$ are interlaced.

For HB, let $p=g+\mathrm{i} f$. Considering i $p=-f+\mathrm{i} g$ if necessary, we can assume that $\operatorname{deg}(f) \leq \operatorname{deg}(g)$. If $f \ll g$, then Exercise 2.5(c) implies that $\operatorname{Im}(f(z) / g(z)) \leq 0$ for all $z \in \mathcal{H}$, so that $g+\mathrm{i} f$ is stable. For the converse, let $p(x)=c \prod_{i=1}^{d}\left(x-\xi_{i}\right)$, so that $\operatorname{Im}\left(\xi_{i}\right) \leq 0$ for all $i \in[d]$. Now $\left|z-\xi_{i}\right| \geq\left|\bar{z}-\xi_{i}\right|$ for all $z \in \mathcal{H}$ and $i \in[d]$, so that $|p(z)| \geq|p(\bar{z})|$ for all $z \in \mathcal{H}$. For any $z \in \mathcal{H}$ with $f(z) \neq 0$, we have

$$
\left|\frac{g(z)}{f(z)}+\mathrm{i}\right| \geq\left|\frac{g(\bar{z})}{f(\bar{z})}+\mathrm{i}\right|=\left|\frac{g(z)}{f(z)}-\mathrm{i}\right|
$$

and it follows that $\operatorname{Im}(g(z) / f(z)) \geq 0$ for all $z \in \mathcal{H}$ with $f(z) \neq 0$. Since $f g$ has simple roots, it follows that $g(x)+y f(x)$ is stable in $\mathfrak{S}[x, y]$. By contraction and specialization, both $f$ and $g$ are real stable. By scaling and specialization, $a f+b g$ is stable for all $a, b \in \mathbb{R}$. By HKO, the roots of $f$ and $g$ are interlaced. Since $\operatorname{Im}(f(z) / g(z)) \leq 0$ for all $z \in \mathcal{H}$, all the $b_{i}$ in Exercise 2.5(c) are positive, so that $\mathrm{W}[f, g]$ is negative on all of $\mathbb{R}$ : that is $f \ll g$.

For $\lambda: \mathbb{N} \rightarrow \mathbb{R}$, let $T_{\lambda}: \mathbb{R}[x] \rightarrow \mathbb{R}[x]$ be the linear transformation defined by $T_{\lambda}\left(x^{n}\right)=\lambda(n) x^{n}$ and linear extension. A multiplier sequence (of the first kind) is such a $\lambda$ for which $T_{\lambda}(f)$ is real stable whenever $f$ is real stable. Pólya and Schur characterized multiplier sequences as follows.

Pólya-Schur Theorem (Theorem 1.7 of [4]). Let $\lambda: \mathbb{N} \rightarrow \mathbb{R}$. The following are equivalent:

(a) $\lambda$ is a multiplier sequence.

(b) $F_{\lambda}(x)=\sum_{n=0}^{\infty} \lambda(n) x^{n} / n$ ! is an entire function which is the limit, uniformly on compact sets, of real stable polynomials with all roots of the same sign. 
(c) Either $F_{\lambda}(x)$ or $F_{\lambda}(-x)$ has the form

$$
C x^{n} \mathrm{e}^{a x} \prod_{j=1}^{\infty}\left(1+\alpha_{j} x\right),
$$

in which $C \in \mathbb{R}, n \in \mathbb{N}, a \geq 0$, all $\alpha_{j} \geq 0$, and $\sum_{j=1}^{\infty} \alpha_{j}$ is finite.

(d) For all $n \in \mathbb{N}$, the polynomial $T_{\lambda}\left((1+x)^{n}\right)$ is real stable with all roots of the same sign.

One of the main results of Borcea and Brändén's theory is a great generalization of the Pólya-Schur Theorem - a characterization of all stability preservers: linear transformations $T: \mathbb{C}[\mathbf{x}] \rightarrow \mathbb{C}[\mathbf{x}]$ such that $T(f)$ is stable whenever $f$ is stable. (Also the analogous characterization of real stability preservers.) This is discussed in some detail in Section 5.3.

2.4. Multivariate analogues of the HKO and HB Theorems. By analogy with the univariate HB Theorem, polynomials $f, g \in \mathbb{R}[\mathbf{x}]$ are said to be in proper position, denoted by $f \ll g$, when $g+\mathrm{i} f \in \mathfrak{S}[\mathbf{x}]$. (As will be seen, this implies that $f, g \in \mathfrak{S}_{\mathbb{R}}[\mathbf{x}]$.) Thus, the multivariate analogue of Hermite-Biehler is a definition, not a theorem.

Proposition 2.7 (Lemma 1.8 and Remark 1.3 of [5]). Let $f, g \in \mathbb{C}[\mathbf{x}]$.

(a) If $f, g \in \mathbb{R}[\mathbf{x}]$, then $f \ll g$ if and only if $g+y f \in \mathfrak{S}_{\mathbb{R}}[\mathbf{x}, y]$.

(b) If $0 \not \equiv f \in \mathfrak{S}[\mathbf{x}]$, then $g+y f \in \mathfrak{S}[\mathbf{x}, y]$ if and only if for all $\mathbf{z} \in \mathcal{H}^{m}$,

$$
\operatorname{Im}\left(\frac{g(\mathbf{z})}{f(\mathbf{z})}\right) \geq 0 \text {. }
$$

Proof. If $g+y f \in \mathfrak{S}_{\mathbb{R}}[\mathbf{x}, y]$, then $g+$ if $\in \mathfrak{S}[\mathbf{x}]$ by specialization. Conversely, assume that $h=g+\mathrm{i} f \in \mathfrak{S}[\mathbf{x}]$ with $f, g \in \mathbb{R}[\mathbf{x}]$, and let $z=a+\mathrm{i} b$ with $a, b \in \mathbb{R}$ and $b>0$. By Lemma 2.3, for all $\mathbf{a}, \mathbf{b} \in \mathbb{R}^{m}$ with $\mathbf{b}>\mathbf{0}$, we have $h(\mathbf{a}+\mathbf{b} t) \in \mathfrak{S}[t]$. By HB, $\widehat{f}(t)=f(\mathbf{a}+\mathbf{b} t)$ and $\widehat{g}(t)=g(\mathbf{a}+\mathbf{b} t)$ are such that $\widehat{f} \ll \widehat{g}$. By HKO, $c \widehat{f}+d \widehat{g} \in \mathfrak{S}_{\mathbb{R}}[t]$ for all $c, d \in \mathbb{R}$. By HKO again, the roots of $b \widehat{f}$ and of $\widehat{g}+a \widehat{f}$ are interlaced. Since $W[b \widehat{f}, \widehat{g}+a \widehat{f}]=b W[\widehat{f}, \widehat{g}] \leq 0$ on $\mathbb{R}$, it follows that $b \widehat{f} \ll \widehat{g}+a \widehat{f}$. Finally, by HB again, $\widehat{g}+(a+\mathrm{i} b) \hat{f} \in \mathfrak{S}[t]$. Since this holds for all $\mathbf{a}, \mathbf{b} \in \mathbb{R}^{m}$ with b $>\mathbf{0}$, Lemma 2.3 implies that $g+(a+\mathrm{i} b) f \in \mathfrak{S}[\mathbf{x}]$. Since this holds for all $a, b \in \mathbb{R}$ with $b>0, g+y f \in \mathfrak{S}[\mathbf{x}, y]$. This proves part (a).

For part (b), first let $g+y f$ be stable. By specialization, $g$ is also stable. If $g \equiv 0$, then there is nothing to prove. Otherwise, consider any $\mathbf{z} \in \mathcal{H}^{m}$, so that $f(\mathbf{z}) \neq 0$ and $g(\mathbf{z}) \neq 0$. There is a unique solution $z \in \mathbb{C}$ to $g(\mathbf{z})+z f(\mathbf{z})=0$, and since $g+y f$ is stable, $\operatorname{Im}(z) \leq 0$. Hence, $\operatorname{Im}(g(\mathbf{z}) / f(\mathbf{z}))=\operatorname{Im}(-z) \geq 0$. This argument can be reversed to prove the converse implication.

Exercise 2.8 (Corollary 2.4 of [4]). $\mathfrak{S}[\mathbf{x}]=\left\{g+\mathrm{i} f: f, g \in \mathfrak{S}_{\mathbb{R}}[\mathbf{x}]\right.$ and $\left.f \ll g\right\}$.

Here is the multivariate HKO Theorem of Borcea and Brändén.

Theorem 2.9 (Theorem 1.6 of $\left[4\right.$ ). Let $f, g \in \mathbb{R}[\mathbf{x}]$. Then af $+b g \in \mathfrak{S}_{\mathbb{R}}[\mathbf{x}]$ for all $a, b \in \mathbb{R}$ if and only if $f, g \in \mathfrak{S}_{\mathbb{R}}[\mathbf{x}]$ and either $f \ll g$ or $g \ll f$.

Proof. First assume that $f \ll g$, and let $a, b \in \mathbb{R}$ with $b>0$. By Proposition 2.7(a), $g+y f \in \mathfrak{S}_{\mathbb{R}}[\mathbf{x}, y]$. By scaling and specialization, $b g+(a+\mathrm{i}) f \in \mathfrak{S}[\mathbf{x}]$. By Proposition 2.7(a) again, $f \ll(a f+b g)$. Thus $a f+b g \in \mathfrak{S}_{\mathbb{R}}[\mathbf{x}]$ for all $a, b \in \mathbb{R}$. The case that $g \ll f$ is similar. 
Conversely, assume that $a f+b g \in \mathfrak{S}_{\mathbb{R}}[\mathbf{x}]$ for all $a, b \in \mathbb{R}$. Let $\mathbf{a}, \mathbf{b} \in \mathbb{R}^{m}$ with $\mathbf{b}>\mathbf{0}$, and let $\widehat{f}(t)=f(\mathbf{a}+\mathbf{b} t)$ and $\widehat{g}(t)=g(\mathbf{a}+\mathbf{b} t)$. By Lemma 2.3, $a \widehat{f}+b \widehat{g} \in \mathfrak{S}_{\mathbb{R}}[t]$ for all $a, b \in \mathbb{R}$. By HKO, for each $\mathbf{a}, \mathbf{b} \in \mathbb{R}^{m}$ with $\mathbf{b}>\mathbf{0}$, either $\widehat{f} \ll \widehat{g}$ or $\widehat{g} \ll \widehat{f}$.

If $\widehat{f} \ll \widehat{g}$ for all $\mathbf{a}, \mathbf{b} \in \mathbb{R}^{m}$ with $\mathbf{b}>\mathbf{0}$, then by $\mathrm{HB}, \widehat{g}+\mathrm{i} \widehat{f} \in \mathfrak{S}[t]$ for all $\mathbf{a}, \mathbf{b} \in \mathbb{R}^{m}$ with $\mathbf{b}>\mathbf{0}$. Thus $g+$ if $\in \mathfrak{S}[\mathbf{x}]$ by Lemma 2.3 , which is to say that $f \ll g$ (by definition). Similarly, if $\widehat{g} \ll \widehat{f}$ for all $\mathbf{a}, \mathbf{b} \in \mathbb{R}^{m}$ with $\mathbf{b}>\mathbf{0}$, then $g \ll f$.

It remains to consider the case that $f\left(\mathbf{a}_{0}+\mathbf{b}_{0} t\right) \ll g\left(\mathbf{a}_{0}+\mathbf{b}_{0} t\right)$ for some $\mathbf{a}_{0}, \mathbf{b}_{0} \in$ $\mathbb{R}^{m}$ with $\mathbf{b}_{0}>\mathbf{0}$, and $g\left(\mathbf{a}_{1}+\mathbf{b}_{1} t\right) \ll f\left(\mathbf{a}_{1}+\mathbf{b}_{1} t\right)$ for another $\mathbf{a}_{1}, \mathbf{b}_{1} \in \mathbb{R}^{m}$ with $\mathbf{b}_{1}>\mathbf{0}$. For $0 \leq \lambda \leq 1$, let $\mathbf{a}_{\lambda}=(1-\lambda) \mathbf{a}_{0}+\lambda \mathbf{a}_{1}$ and $\mathbf{b}_{\lambda}=(1-\lambda) \mathbf{b}_{0}+\lambda \mathbf{b}_{1}$. Since roots of polynomials move continuously as the coefficients are varied continuously, there is a value $0 \leq \lambda \leq 1$ for which both $f\left(\mathbf{a}_{\lambda}+\mathbf{b}_{\lambda} t\right) \ll g\left(\mathbf{a}_{\lambda}+\mathbf{b}_{\lambda} t\right)$ and $g\left(\mathbf{a}_{\lambda}+\mathbf{b}_{\lambda} t\right) \ll f\left(\mathbf{a}_{\lambda}+\mathbf{b}_{\lambda} t\right)$. From Exercise 2.6, it follows that $c f\left(\mathbf{a}_{\lambda}+\mathbf{b}_{\lambda} t\right)=$ $d g\left(\mathbf{a}_{\lambda}+\mathbf{b}_{\lambda} t\right)$ for some $c, d \in \mathbb{R}$ not both zero. Now $h=c f-d g \in \mathfrak{S}[\mathbf{x}]$ by hypothesis, and since $h\left(\mathbf{a}_{\lambda}+\mathbf{b}_{\lambda} t\right) \equiv 0$ identically, it follows that $h\left(\mathbf{a}_{\lambda}+\mathrm{i} \mathbf{b}_{\lambda}\right)=0$. Since $\mathbf{b}_{\lambda}>\mathbf{0}$ and $h$ is stable, this implies that $h \equiv 0$, so that $c f=d g$ in $\mathfrak{S}[\mathbf{x}]$. In this case, both $f \ll g$ and $g \ll f$ hold.

For $f, g \in \mathbb{C}[\mathbf{x}]$ and $i \in[m]$, let $\mathrm{W}_{i}[f, g]=\partial_{i} f \cdot g-f \cdot \partial_{i} g$ be the $i$ th Wronskian of the pair $(f, g)$.

Corollary $\mathbf{2 . 1 0}$ (Theorem 1.9 of [5]). Let $f, g \in \mathbb{R}[\mathbf{x}]$. The following are equivalent:

(a) $g+$ if is stable in $\mathfrak{S}[\mathbf{x}]$, that is $f \ll g$;

(b) $g+y f$ is real stable in $\mathfrak{S}_{\mathbb{R}}[\mathbf{x}, y]$;

(c) af $+b g \in \mathfrak{S}_{\mathbb{R}}[\mathbf{x}]$ for all $a, b \in \mathbb{R}$, and $\mathrm{W}_{i}[f, g](\mathbf{a}) \leq 0$ for all $i \in[m]$ and $\mathbf{a} \in \mathbb{R}^{m}$.

Proof. Proposition 2.7(a) shows that (a) and (b) are equivalent.

If (a) holds, then Theorem 2.9 implies that $a f+b g \in \mathfrak{S}_{\mathbb{R}}[\mathbf{x}]$ for all $a, b \in \mathbb{R}$. To prove the rest of (c), let $i \in[m]$ and $\mathbf{a} \in \mathbb{R}^{m}$, and let $\delta_{i} \in \mathbb{R}^{m}$ be the unit vector with a one in the $i$ th position. Since $f \ll g$, for any $\mathbf{b} \in \mathbb{R}^{m}$ with $\mathbf{b}>\mathbf{0}$ we have $f\left(\mathbf{a}+\left(\mathbf{b}+\delta_{i}\right) t\right) \ll g\left(\mathbf{a}+\left(\mathbf{b}+\delta_{i}\right) t\right)$ in $\mathfrak{S}_{\mathbb{R}}[t]$, from Proposition 2.7(a) and Lemma 2.3. By the Wronskian condition for univariate polynomials in proper position,

$$
\mathrm{W}\left[f\left(\mathbf{a}+\left(\mathbf{b}+\delta_{i}\right) t\right), g\left(\mathbf{a}+\left(\mathbf{b}+\delta_{i}\right) t\right)\right] \leq 0
$$

for all $t \in \mathbb{R}$. Taking the limit as $\mathbf{b} \rightarrow \mathbf{0}$ and evaluating at $t=0$ yields

$$
\mathrm{W}_{i}[f, g](\mathbf{a})=\left.\mathrm{W}\left[f\left(\mathbf{a}+\delta_{i} t\right), g\left(\mathbf{a}+\delta_{i} t\right)\right]\right|_{t=0} \leq 0
$$

by continuity. Thus (a) implies (c).

To prove that (c) implies (b), let $\mathbf{a}, \mathbf{b} \in \mathbb{R}^{m}$ with $\mathbf{b}=\left(b_{1}, \ldots, b_{m}\right)>\mathbf{0}$, and let $a, b \in \mathbb{R}$ with $b>0$. By Lemma 2.3 , to show that $g+y f \in \mathfrak{S}_{\mathbb{R}}[\mathbf{x}, y]$, it suffices to show that $g(\mathbf{a}+\mathbf{b} t)+(a+\mathrm{i} b) f(\mathbf{a}+\mathbf{b} t) \in \mathfrak{S}[t]$. From (c) it follows that $p=g+a f$ and $q=b f$ are such that $\alpha p+\beta q \in \mathfrak{S}_{\mathbb{R}}[\mathbf{x}]$ for all $\alpha, \beta \in \mathbb{R}$. By Theorem 2.9, either $p \ll q$ or $q \ll p$. Now

$$
\begin{aligned}
\mathrm{W}[q(\mathbf{a}+\mathbf{b} t), p(\mathbf{a}+\mathbf{b} t)] & =b \mathrm{~W}[f(\mathbf{a}+\mathbf{b} t), g(\mathbf{a}+\mathbf{b} t)] \\
& =b \sum_{i=1}^{m} b_{i} \mathrm{~W}_{i}[f, g](\mathbf{a}+\mathbf{b} t) \leq 0,
\end{aligned}
$$

by the Wronskian condition in part (c). Thus, $q(\mathbf{a}+\mathbf{b} t) \ll p(\mathbf{a}+\mathbf{b} t)$, so that $p(\mathbf{a}+\mathbf{b} t)+\mathrm{i} q(\mathbf{a}+\mathbf{b} t) \in \mathfrak{S}[t]$. Since $p+\mathrm{i} q=g+(a+\mathrm{i} b) f$, this shows that (c) implies (b). 
Exercise 2.11 (Corollary 1.10 of $[5]$ ). Let $f, g \in \mathfrak{S}_{\mathbb{R}}[\mathbf{x}]$ be real stable. Then $f \ll g$ and $g \ll f$ if and only if $c f=d g$ for some $c, d \in \mathbb{R}$ not both zero.

Proposition 2.12 (Lemma 3.2 of $[5]$ ). Let $V$ be a $\mathbb{K}$-vector subspace of $\mathbb{K}[\mathbf{x}]$, with either $\mathbb{K}=\mathbb{R}$ or $\mathbb{K}=\mathbb{C}$.

(a) If $\mathbb{K}=\mathbb{R}$ and $V \subseteq \mathfrak{S}_{\mathbb{R}}[\mathbf{x}]$, then $\operatorname{dim}_{\mathbb{R}} V \leq 2$.

(b) If $\mathbb{K}=\mathbb{C}$ and $V \subseteq \mathfrak{S}[\mathbf{x}]$, then $\operatorname{dim}_{\mathbb{C}} V \leq 1$.

Proof. For part (a), suppose to the contrary that $f, g, h \in V$ are linearly independent over $\mathbb{R}$ (and hence not identically zero). By Theorem 2.9, either $f \ll g$ or $g \ll f$, and similarly for the other pairs $\{f, h\}$ and $\{g, h\}$. Renaming these polynomials as necessary, we may assume that $f \ll h$ and $h \ll g$. Now, for all $\lambda \in[0,1]$, let $p_{\lambda}=(1-\lambda) f+\lambda g$, and note that each $p_{\lambda} \not \equiv 0$. By Theorem 2.9, for each $\lambda \in[0,1]$ either $h \ll p_{\lambda}$ or $p_{\lambda} \ll h$. Since $p_{0}=f \ll h$ and $h \ll g=p_{1}$, by continuity of the roots of $\left\{p_{\lambda}: \lambda \in[0,1]\right\}$ there is a $\lambda \in[0,1]$ such that $h \ll p_{\lambda}$ and $p_{\lambda} \ll h$. But then, by Exercise 2.11, either $\{f, g\}$ is linearly dependent or $h$ is in the span of $\{f, g\}$, contradicting the supposition.

For part (b), let $\operatorname{Re}(V)=\{\operatorname{Re}(h): h \in V\}$. Then $\operatorname{Re}(V)$ is a real subspace of $\mathfrak{S}_{\mathbb{R}}[\mathbf{x}]$, so that $\operatorname{dim}_{\mathbb{R}} \operatorname{Re}(V) \leq 2$ by part (a). If $\operatorname{dim}_{\mathbb{R}} \operatorname{Re}(V) \leq 1$, then $\operatorname{dim}_{\mathbb{C}} V \leq 1$. In the remaining case let $\{p, q\}$ be a basis of $\operatorname{Re}(V)$ with $f=p+\mathrm{i} q \in V$. By Corollary 2.10, $\mathrm{W}_{i}[q, p](\mathbf{a}) \leq 0$ for all $i \in[m]$ and $\mathbf{a} \in \mathbb{R}^{m}$. Since $p$ and $q$ are not linearly dependent, there is an index $k \in[m]$ such that $\mathrm{W}_{k}[q, p] \not \equiv 0$.

Consider any $g \in V$. There are reals $a, b, c, d \in \mathbb{R}$ such that

$$
g=(a p+b q)+\mathrm{i}(c p+d q) .
$$

Since $g$ is stable, $\mathrm{W}_{k}[c p+d q, a p+b q](\mathbf{a})=(a d-b c) \mathrm{W}_{k}[q, p](\mathbf{a}) \leq 0$ for all $\mathbf{a} \in \mathbb{R}^{m}$. Since $\mathrm{W}_{k}[q, p] \not \equiv 0$, it follows that $a d-b c \geq 0$. Now, for any $v, w \in \mathbb{R}, g+(v+\mathrm{i} w) f$ is in $V$. Since

$$
g+(v+\mathrm{i} w) f=(a+v) p+(b-w) q+\mathrm{i}((c+w) p+(d+v) q),
$$

this argument shows that $H=(a+v)(d+v)-(b-w)(c+w) \geq 0$ for all $v, w \in \mathbb{R}$. But

$$
4 H=(2 v+a+d)^{2}+(2 w+c-b)^{2}-(a-d)^{2}-(b+c)^{2},
$$

so that $H \geq 0$ for all $v, w \in \mathbb{R}$ if and only if $a=d$ and $b=-c$. This implies that $g=(a+\mathrm{i} c) f$, so that $\operatorname{dim}_{\mathbb{C}} V=1$.

\section{Multiaffine stable Polynomials}

A polynomial $f$ is multiaffine if each indeterminate occurs at most to the first power in $f$. For a set $\mathcal{S}$ of polynomials, let $\mathcal{S}^{\mathrm{MA}}$ denote the set of multiaffine polynomials in $\mathcal{S}$. For multiaffine $f \in \mathbb{C}[\mathbf{x}]^{\mathrm{MA}}$ and $i \in[m]$ we use the "ultrashorthand" notation $f=f^{i}+x_{i} f_{i}$, in which $f^{i}=\left.f\right|_{x_{i}=0}$ and $f_{i}=\partial_{i} f$. This notation is extended to multiple distinct indices in the obvious way - in particular,

$$
f=f^{i j}+x_{i} f_{i}^{j}+x_{j} f_{j}^{i}+x_{i} x_{j} f_{i j} .
$$

3.1. A criterion for real stability. For $f \in \mathbb{C}[\mathbf{x}]$ and $\{i, j\} \subseteq[m]$, let

$$
\Delta_{i j} f=\partial_{i} f \cdot \partial_{j} f-f \cdot \partial_{i} \partial_{j} f .
$$

Notice that for $f \in \mathbb{C}[\mathbf{x}]^{\mathrm{MA}}$,

$$
\Delta_{i j} f=f_{i}^{j} f_{j}-f^{j} f_{i j}=\mathrm{W}_{i}\left[f^{j}, f_{j}\right]=-\mathrm{W}_{i}\left[f_{j}, f^{j}\right]
$$

and

$$
\Delta_{i j} f=f_{i}^{j} f_{j}^{i}-f^{i j} f_{i j}
$$


Theorem 3.1 (Theorem 5.6 of $\left[8\right.$ and Theorem 3 of $[19]$ ). Let $f \in \mathbb{R}[\mathbf{x}]^{\mathrm{MA}}$ be multiaffine. The following are equivalent:

(a) $f$ is real stable.

(b) For all $\{i, j\} \subseteq[m]$ and all $\mathbf{a} \in \mathbb{R}^{m}, \Delta_{i j} f(\mathbf{a}) \geq 0$.

(c) Either $m=1$, or there exists $\{i, j\} \subseteq[m]$ such that $f_{i}, f^{i}, f_{j}$, and $f^{j}$ are real stable, and $\Delta_{i j} f(\mathbf{a}) \geq 0$ for all $\mathbf{a} \in \mathbb{R}^{m}$.

Proof. To see that (a) implies (b), fix $\{i, j\} \subseteq[m]$. Proposition 2.7(a) shows that $f_{j} \ll f^{j}$, and from the calculation above and Corollary 2.10, it follows that $\Delta_{i j} f(\mathbf{a})=-\mathrm{W}_{i}\left[f_{j}, f^{j}\right](\mathbf{a}) \geq 0$ for all $\mathbf{a} \in \mathbb{R}^{m}$.

We show that (b) implies (a) by induction on $m$, the base case $m=1$ being trivial. For the induction step let $f$ be as in part (b), let $a \in \mathbb{R}$, and let $g=$ $\left.f\right|_{x_{m}=a}$. For all $\{i, j\} \subseteq[m-1]$ and $\mathbf{a} \in \mathbb{R}^{m-1}, \Delta_{i j} g(\mathbf{a})=\Delta_{i j} f(\mathbf{a}, a) \geq 0$. By induction, $g=f^{m}+a f_{m}$ is real stable for all $a \in \mathbb{R}$; it follows that $a f_{m}+b f^{m} \in$ $\mathfrak{S}_{\mathbb{R}}\left[x_{1}, \ldots, x_{m-1}\right]$ for all $a, b \in \mathbb{R}$. Furthermore, for all $j \in[m-1]$ and $\mathbf{a} \in \mathbb{R}^{m-1}$, $\mathrm{W}_{j}\left[f_{m}, f^{m}\right](\mathbf{a})=-\Delta_{j m} f(\mathbf{a}, 1) \leq 0$. This verifies condition (c) of Corollary 2.10 for the pair $\left(f_{m}, f^{m}\right)$, and it follows that $f=f^{m}+x_{m} f_{m} \in \mathfrak{S}_{\mathbb{R}}[\mathbf{x}]$, completing the induction.

It is clear that (a) and (b) imply (c) - we show that (c) implies (b) below. This is clear if $m \leq 2$, so assume that $m \geq 3$. To begin with, let $\{h, i, j\} \subseteq[m]$ be three distinct indices, and consider $\Delta_{i j} f$ as a polynomial in $x_{h}$. That is, $\Delta_{i j} f=$ $A_{h i j} x_{h}^{2}+B_{h i j} x_{h}+C_{h i j}$ in which

$$
\begin{aligned}
A_{h i j} & =f_{h i}^{j} f_{h j}^{i}-f_{h}^{i j} f_{h i j}=\Delta_{i j} f_{h}, \\
B_{h i j} & =f_{h i}^{j} f_{j}^{h i}-f_{h}^{i j} f_{i j}^{h}+f_{i}^{h j} f_{h j}^{i}-f^{h i j} f_{h i j}, \text { and } \\
C_{h i j} & =f_{i}^{h j} f_{j}^{h i}-f^{h i j} f_{i j}^{h}=\Delta_{i j} f^{h} .
\end{aligned}
$$

If $\Delta_{i j} f(\mathbf{a}) \geq 0$ for all $\mathbf{a} \in \mathbb{R}^{m}$, then this quadratic polynomial in $x_{h}$,

$$
\Delta_{i j} f\left(a_{1}, \ldots, a_{h-1}, x_{h}, a_{h+1}, \ldots, a_{m}\right),
$$

has a nonpositive discriminant for all $\mathbf{a} \in \mathbb{R}^{m}$. That is, $D_{h i j}=B_{h i j}^{2}-4 A_{h i j} C_{h i j}$ is such that $D_{h i j}(\mathbf{a}) \leq 0$ for all $\mathbf{a} \in \mathbb{R}^{m}$.

It is a surprising fact that as a polynomial in $\left\{x_{k}: k \in[m] \backslash\{h, i, j\}\right\}, D_{h i j}$ is invariant under all six permutations of its indices, as is seen by direct calculation:

$$
\begin{aligned}
D_{h i j}= & \left(f_{h}^{i j} f_{i j}^{h}\right)^{2}+\left(f_{i}^{h j} f_{h j}^{i}\right)^{2}+\left(f_{j}^{h i} f_{h i}^{j}\right)^{2}+\left(f_{h i j} f^{h i j}\right)^{2} \\
& -2\left(f_{h}^{i j} f_{i j}^{h} f_{i}^{h j} f_{h j}^{i}+f_{i}^{h j} f_{h j}^{i} f_{j}^{h i} h_{h i}^{j}+f_{j}^{h i} f_{h i}^{j} f_{h}^{i j} f_{i j}^{h}\right) \\
& -2\left(f_{h}^{i j} f_{i j}^{h}+f_{i}^{h j} f_{h j}^{i}+f_{j}^{h i} f_{h i}^{j}\right) f^{h i j} f_{h i j} \\
& +4 f_{i j}^{h} f_{h j}^{i} f_{h i}^{j} f^{h i j}+4 f_{h}^{i j} f_{i}^{h j} f_{j}^{h i} f_{h i j} .
\end{aligned}
$$

Now for the proof that (c) implies (b) when $m \geq 3$ : Consider any $h \in[m] \backslash\{i, j\}$. Then

$$
\Delta_{h i} f=A_{j h i} x_{j}^{2}+B_{j h i} x_{j}+C_{j h i}
$$

has discriminant $D_{j h i}=D_{h i j}$. Since $f_{j}$ and $f^{j}$ are real stable, we have $A_{j h i}(\mathbf{a})=$ $\Delta_{h i} f_{j}(\mathbf{a}) \geq 0$ and $C_{j h i}(\mathbf{a})=\Delta_{h i} f^{j}(\mathbf{a}) \geq 0$ for all $\mathbf{a} \in \mathbb{R}^{m}$. Since $\Delta_{i j} f(\mathbf{a}) \geq 0$ for all $\mathbf{a} \in \mathbb{R}^{m}$ it follows that $D_{j h i}(\mathbf{a})=D_{h i j}(\mathbf{a}) \leq 0$ for all $\mathbf{a} \in \mathbb{R}^{m}$. It follows that $\Delta_{h i} f(\mathbf{a}) \geq 0$ for all $\mathbf{a} \in \mathbb{R}^{m}$. (Note that if $B^{2}-4 A C \leq 0$ and either $A=0$ or $C=0$, then $B=0$.) A similar argument using the fact that $f_{i}$ and $f^{i}$ are real stable shows that $\Delta_{h j} f(\mathbf{a}) \geq 0$ for all $\mathbf{a} \in \mathbb{R}^{m}$. 
It remains to show that $\Delta_{h k} f(\mathbf{a}) \geq 0$ for all $\mathbf{a} \in \mathbb{R}^{m}$ when $\{h, k\}$ is disjoint from $\{i, j\}$. We have seen that $\Delta_{h i} f(\mathbf{a}) \geq 0$ for all $\mathbf{a} \in \mathbb{R}^{m}$, and we know that both $f_{i}$ and $f^{i}$ are real stable. The argument above applies once more: $\Delta_{h i} f(\mathbf{a}) \geq 0$ for all $\mathbf{a} \in \mathbb{R}^{m}$, so that $D_{i h k}(\mathbf{a})=D_{k h i}(\mathbf{a}) \leq 0$ for all $\mathbf{a} \in \mathbb{R}^{m}$, and then since $A_{i h k}(\mathbf{a}) \geq 0$ and $C_{i h k}(\mathbf{a}) \geq 0$ for all $\mathbf{a} \in \mathbb{R}^{m}$ it follows that $\Delta_{h k} f(\mathbf{a}) \geq 0$ for all $\mathbf{a} \in \mathbb{R}^{m}$. Thus (c) implies (b).

\subsection{Linear transformations preserving stability: multiaffine case.}

Lemma 3.2 (Lieb-Sokal Lemma, Lemma 2.1 of [5]). Let $g(\mathbf{x})+y f(\mathbf{x}) \in \mathfrak{S}[\mathbf{x}, y]$ be stable and such that $\operatorname{deg}_{i}(f) \leq 1$. Then $g-\partial_{i} f \in \mathfrak{S}[\mathbf{x}]$ is stable.

Proof. Since $g$ is stable (by specialization to $y=0$ ), there is nothing to prove if $\partial_{i} f \equiv 0$ identically, so assume otherwise (and hence that $f \not \equiv 0$ ). By permutation we can assume that $i=1$. Since $f$ is stable and $z_{1}, z \in \mathcal{H}$ imply that $z_{1}-z^{-1} \in \mathcal{H}$, it follows that

$$
y f\left(x_{1}-y^{-1}, x_{2}, \ldots, x_{m}\right)=-\partial_{1} f(\mathbf{x})+y f(\mathbf{x})
$$

is stable. Proposition 2.7(b) implies that for all $\mathbf{z} \in \mathcal{H}^{m}$,

$$
\operatorname{Im}\left(\frac{g(\mathbf{z})-\partial_{1} f(\mathbf{z})}{f(\mathbf{z})}\right)=\operatorname{Im}\left(\frac{g(\mathbf{z})}{f(\mathbf{z})}\right)+\operatorname{Im}\left(\frac{-\partial_{1} f(\mathbf{z})}{f(\mathbf{z})}\right) \geq 0 .
$$

Thus, by Proposition 2.7(b) again, $g-\partial_{1} f+y f$ is stable. Specializing to $y=0$ shows that $g-\partial_{1} f$ is stable.

Exercise 3.3 (Lemma 3.1 of $[5]$ ). Let $f \in \mathbb{C}[\mathbf{x}]^{\mathrm{MA}}$ and $\mathbf{w} \in \mathcal{H}^{m}$. Then for all $\epsilon>0$ sufficiently small, $(\mathbf{x}+\mathbf{w})^{[m]}+\epsilon f(\mathbf{x})$ is stable. $\left(\right.$ Here $(\mathbf{x}+\mathbf{w})^{[m]}=\prod_{i=1}^{m}\left(x_{i}+w_{i}\right)$.)

For a linear transformation $T: \mathbb{C}[\mathbf{x}]^{\mathrm{MA}} \rightarrow \mathbb{C}[\mathbf{x}]$ of multiaffine polynomials, define the algebraic symbol of $T$ to be the polynomial

$$
T\left((\mathbf{x}+\mathbf{y})^{[m]}\right)=T\left(\prod_{i=1}^{m}\left(x_{i}+y_{i}\right)\right)=\sum_{S \subseteq[m]} T\left(\mathbf{x}^{S}\right) \mathbf{y}^{[m] \backslash S}
$$

in $\mathbb{C}\left[x_{1}, \ldots, x_{m}, y_{1}, \ldots, y_{m}\right]=\mathbb{C}[\mathbf{x}, \mathbf{y}]$.

Theorem 3.4 (Theorem 1.1 of $[5]$ ). Let $T: \mathbb{C}[\mathbf{x}]^{\mathrm{MA}} \rightarrow \mathbb{C}[\mathbf{x}]$ be a linear transformation. Then $T$ maps $\mathfrak{S}[\mathbf{x}]^{\mathrm{MA}}$ into $\mathfrak{S}[\mathbf{x}]$ if and only if either

(a) $T(f)=\eta(f) \cdot p$ for some linear functional $\eta: \mathbb{C}[\mathbf{x}]^{\mathrm{MA}} \rightarrow \mathbb{C}$ and $p \in \mathfrak{S}[\mathbf{x}]$, or

(b) the polynomial $T\left((\mathbf{x}+\mathbf{y})^{[m]}\right)$ is stable in $\mathfrak{S}[\mathbf{x}, \mathbf{y}]$.

Proof. First, assume (b) that $T\left((\mathbf{x}+\mathbf{y})^{[m]}\right) \in \mathfrak{S}[\mathbf{x}, \mathbf{y}]$ is stable. By inversion, it follows that $\mathbf{y}^{[m]} T\left(\left(\mathbf{x}-\mathbf{y}^{-\mathbf{1}}\right)^{[m]}\right)$ is also stable. Thus, if $f \in \mathfrak{S}\left[w_{1}, \ldots, w_{m}\right]$ is stable, then

$$
\mathbf{y}^{[m]} T\left(\left(\mathbf{x}-\mathbf{y}^{-\mathbf{1}}\right)^{[m]}\right) f(\mathbf{w})=\sum_{S \subseteq[m]} T\left(\mathbf{x}^{S}\right)(-\mathbf{y})^{S} f(\mathbf{w})
$$

is stable. If $f$ is also multiaffine, then repeated application of the Lieb-Sokal Lemma 3.2 (replacing $y_{i}$ by $-\partial / \partial w_{i}$ for $i \in[m]$ ) shows that

$$
\sum_{S \subseteq[m]} T\left(\mathbf{x}^{S}\right) \frac{\partial^{S}}{\partial \mathbf{w}^{S}} f(\mathbf{w})
$$

is stable. Finally, specializing to $\mathbf{w}=\mathbf{0}$ shows that $T(f(\mathbf{x}))$ is stable. Thus, the linear transformation $T$ maps $\mathfrak{S}[\mathbf{x}]^{\mathrm{MA}}$ into $\mathfrak{S}[\mathbf{x}]$. This is clearly also the case if (a) holds. 
Conversely, assume that $T$ maps $\mathfrak{S}[\mathbf{x}]^{\mathrm{MA}}$ into $\mathfrak{S}[\mathbf{x}]$. Then for any $\mathbf{w} \in \mathcal{H}^{m}$, $(\mathbf{x}+\mathbf{w})^{[m]} \in \mathfrak{S}[\mathbf{x}]^{\mathrm{MA}}$, so that $T\left((\mathbf{x}+\mathbf{w})^{[m]}\right) \in \mathfrak{S}[\mathbf{x}]$.

First, assume that there is a $\mathbf{w} \in \mathcal{H}^{m}$ for which $T\left((\mathbf{x}+\mathbf{w})^{[m]}\right) \equiv 0$ identically. For any $f \in \mathbb{C}[\mathbf{x}]^{\mathrm{MA}}$, let $\epsilon>0$ be as in Exercise 3.3. Then $\epsilon T(f)=T\left((\mathbf{x}+\mathbf{w})^{[m]}+\epsilon f\right)$ is stable, so that $T(f)$ is stable. Thus, the image of $\mathbb{C}[\mathbf{x}]^{\mathrm{MA}}$ under $T$ is a $\mathbb{C}$-subspace of $\mathfrak{S}[\mathbf{x}]$. By Proposition 2.12(b), $T$ has the form of case (a).

Secondly, if $T\left((\mathbf{x}+\mathbf{w})^{[m]}\right) \not \equiv 0$ for all $\mathbf{w} \in \mathcal{H}^{m}$, then, since each of these polynomials is in $\mathfrak{S}[\mathbf{x}]$, we have $\left.T\left((\mathbf{x}+\mathbf{w})^{[m]}\right)\right|_{\mathbf{x}=\mathbf{z}} \neq 0$ for all $\mathbf{z} \in \mathcal{H}^{m}$ and $\mathbf{w} \in \mathcal{H}^{m}$. This shows that $T\left((\mathbf{x}+\mathbf{y})^{[m]}\right)$ is stable in $\mathfrak{S}[\mathbf{x}, \mathbf{y}]$, which is the form of case (b).

Theorem 3.4 has a corresponding real form; the proof is completely analogous.

Theorem 3.5 (Theorem 1.2 of $[\underline{5})$. Let $T: \mathbb{R}[\mathbf{x}]^{\mathrm{MA}} \rightarrow \mathbb{R}[\mathbf{x}]$ be a linear transformation. Then $T$ maps $\mathfrak{S}_{\mathbb{R}}[\mathbf{x}]^{\mathrm{MA}}$ into $\mathfrak{S}_{\mathbb{R}}[\mathbf{x}]$ if and only if either

(a) $T(f)=\eta(f) \cdot p+\xi(f) \cdot q$ for some linear functionals $\eta, \xi: \mathbb{R}[\mathbf{x}]^{\mathrm{MA}} \rightarrow \mathbb{R}$ and $p, q \in \mathfrak{S}_{\mathbb{R}}[\mathbf{x}]$ such that $p \ll q$, or

(b) the polynomial $T\left((\mathbf{x}+\mathbf{y})^{[m]}\right)$ is real stable in $\mathfrak{S}_{\mathbb{R}}[\mathbf{x}, \mathbf{y}]$, or

(c) the polynomial $T\left((\mathbf{x}-\mathbf{y})^{[m]}\right)$ is real stable in $\mathfrak{S}_{\mathbb{R}}[\mathbf{x}, \mathbf{y}]$.

\section{Proof. Exercise 3.6.}

\section{The Grace-Walsh-Szegő Coincidence Theorem}

Let $f \in \mathbb{C}[x]$ be a univariate polynomial of degree at most $m$, and let $\mathbf{x}=$ $\left(x_{1}, \ldots, x_{m}\right)$ as usual. For $0 \leq j \leq m$, the $j$ th elementary symmetric function of $\mathbf{x}$ is

$$
e_{j}(\mathbf{x})=\sum_{1 \leq i_{1}<\cdots<i_{j} \leq m} x_{i_{1}} \cdots x_{i_{j}}=\sum_{S \subseteq[m]:|S|=j} \mathbf{x}^{S} .
$$

The mth polarization of $f$ is the polynomial obtained as the image of $f$ under the linear transformation $\mathrm{Pol}_{m}$ defined by $x^{j} \mapsto\left(\begin{array}{c}m \\ j\end{array}\right)^{-1} e_{j}(\mathbf{x})$ for all $0 \leq j \leq m$, and linear extension. In other words, $\operatorname{Pol}_{m} f$ is the unique multiaffine polynomial in $\mathbb{C}[\mathbf{x}]^{\mathrm{MA}}$ that is invariant under all permutations of $[\mathrm{m}]$ and such that $\operatorname{Pol}_{m} f(x, \ldots, x)=f(x)$. A circular region is a nonempty subset $\mathcal{A}$ of $\mathbb{C}$ that is either open or closed, and which is bounded by either a circle or a straight line.

Grace-Walsh-Szegö (GWS) Theorem (Theorem 3.4.1b of [16]). Let $f \in \mathbb{C}[x]$ have degree at most $m$, and let $\mathcal{A}$ be a circular region. If either $\operatorname{deg}(f)=m$ or $\mathcal{A}$ is convex, then for every $\mathbf{z} \in \mathcal{A}^{m}$ there exists $z \in \mathcal{A}$ such that $\operatorname{Pol}_{m} f(\mathbf{z})=f(z)$.

The proof of GWS in this section is adapted from Borcea and Brändén [6].

4.1. Reduction to the case of stable polynomials. First of all, it suffices to prove GWS for open circular regions, since a closed circular region is the intersection of all the open circular regions which contain it. Second, it suffices to show that for any $g \in \mathbb{C}[x]$ of degree at most $m$, if $\operatorname{deg}(g)=m$ or $\mathcal{A}$ is convex, and $\mathbf{z} \in \mathcal{A}^{m}$ is such that $\operatorname{Pol}_{m} g(\mathbf{z})=0$, then there exists $z \in \mathcal{A}$ such that $g(z)=0$. This implies the stated form of GWS by applying this special case to $g(x)=f(x)-c$, where $c=\operatorname{Pol}_{m} f(\mathbf{z})$. Stated otherwise, it suffices to show that if $f(z) \neq 0$ for all $z \in \mathcal{A}$ then $\operatorname{Pol}_{m} f(\mathbf{z}) \neq 0$ for all $\mathbf{z} \in \mathcal{A}^{m}$ (provided that either $\operatorname{deg}(f)=m$ or $\mathcal{A}$ is convex).

Let $\mathcal{M}$ be the set of Möbius transformations $z \mapsto \phi(z)=(a z+b) /(c z+d)$ with $a, b, c, d \in \mathbb{C}$ and $a b-c d \neq 0$. Then $\mathcal{M}$ with the operation of functional composition is a group of conformal transformations of the Riemann sphere $\widehat{\mathbb{C}}=\mathbb{C} \cup\{\infty\}$, 
and it acts simply transitively on the set of all ordered triples of distinct points of $\widehat{\mathbb{C}}$. Consequently, for any open circular region $\mathcal{A}$ there is a $\phi \in \mathcal{M}$ such that $\phi(\mathcal{H})=\{\phi(z): z \in \mathcal{H}\}=\mathcal{A}$. We henceforth regard circular regions as subsets of $\widehat{\mathbb{C}}$. Note that an open circular region $\mathcal{A}$ is convex if and only if it does not contain $\infty$. (The point $\infty$ is on the boundary of any open half-plane.) In this case, if $\phi(z)=(a z+b) /(c z+d)$ is such that $\phi(\mathcal{H})=\mathcal{A}$, then $c z+d \neq 0$ for all $z \in \mathcal{H}$.

Given $0 \not \equiv f \in \mathbb{C}[x]$ of degree at most $m$, consider the polynomial $\tilde{f}(x)=$ $(c x+d)^{m} f((a x+b) /(c x+d))$. If either $\operatorname{deg}(f)=m$ or $\mathcal{A}$ is convex, then $f$ is nonvanishing on $\mathcal{A}$ if and only if $\widetilde{f}(z)$ is nonvanishing on $\mathcal{H}$. Also,

$$
\operatorname{Pol}_{m} \tilde{f}(\mathbf{x})=\operatorname{Pol}_{m} f\left(\phi\left(x_{1}\right), \ldots, \phi\left(x_{m}\right)\right) \cdot \prod_{i=1}^{m}\left(c x_{i}+d\right) .
$$

Thus, to prove GWS it suffices to prove the following lemma.

Lemma 4.1. Let $f \in \mathbb{C}[x]$ be a univariate polynomial of degree at most $m$. Then $\mathrm{Pol}_{m} f$ is stable if and only if $f$ is stable.

Clearly, diagonalization implies that if $\mathrm{Pol}_{m} f$ is stable, then $f$ is stable, so only the converse implication needs proof. This is accomplished in the following two easy steps.

4.2. Partial symmetrization. The group $\mathcal{S}(m)$ of all permutations $\sigma:[m] \rightarrow[m]$ acts on $\mathbb{C}[\mathbf{x}]$ by the rule $\sigma(f)\left(x_{1}, \ldots, x_{m}\right)=f\left(x_{\sigma(1)}, \ldots, x_{\sigma(m)}\right)$. Notice that

$$
\sigma\left(\mathbf{x}^{\alpha}\right)=\prod_{i=1}^{m} x_{\sigma(i)}^{\alpha(i)}=\prod_{i=1}^{m} x_{i}^{\alpha \circ \sigma^{-1}(i)}=\mathbf{x}^{\alpha \circ \sigma^{-1}} .
$$

For $\{i, j\} \subseteq[m]$, let $\tau_{i j}$ be the transposition that exchanges $i$ and $j$ and fixes all other elements of $[m]$.

Lemma 4.2. Let $0 \leq \lambda \leq 1$ and $\{i, j\} \subseteq[m]$, and let $T_{i j}^{(\lambda)}=(1-\lambda)+\lambda \tau_{i j}$. If $f \in \mathfrak{S}[\mathbf{x}]^{\mathrm{MA}}$ is stable and multiaffine, then $T_{i j}^{(\lambda)} f \in \mathfrak{S}[\mathbf{x}]^{\mathrm{MA}}$ is stable and multiaffine.

Proof. If $f$ is multiaffine, then $(1-\lambda) f+\lambda \tau_{i j}(f)$ is also multiaffine. We apply Theorem 3.4 to show that $T=T_{i j}^{(\lambda)}$ preserves stability of multiaffine polynomials. By permutation we can assume that $\{i, j\}=\{1,2\}$. The algebraic symbol of $T$ is

$$
T\left((\mathbf{x}+\mathbf{y})^{[m]}\right)=T\left(\left(x_{1}+y_{1}\right)\left(x_{2}+y_{2}\right)\right) \cdot \prod_{i=3}^{m}\left(x_{i}+y_{i}\right) .
$$

Clearly, this is stable if and only if the same is true of $T\left(\left(x_{1}+y_{1}\right)\left(x_{2}+y_{2}\right)\right)$. Exercise 4.3 completes the proof.

Exercise 4.3. Use the results of Sections 2.4 or 3.1 to show that for $0 \leq \lambda \leq 1$, the polynomial

$$
x_{1} x_{2}+\left((1-\lambda) x_{1}+\lambda x_{2}\right) y_{2}+\left(\lambda x_{1}+(1-\lambda) x_{2}\right) y_{1}+y_{1} y_{2}
$$

is real stable. 
4.3. Convergence to the polarization. Let $0 \not \equiv f(x) \in \mathfrak{S}[x]$ be a univariate stable polynomial of degree at most $m$ : say $f(x)=c\left(x-\xi_{1}\right) \cdots\left(x-\xi_{n}\right)$ in which $c \neq 0, n \leq m$, and $\xi_{i} \notin \mathcal{H}$ for all $i \in[n]$. Then the polynomial $F_{0} \in \mathbb{C}[\mathbf{x}]$, defined by

$$
F_{0}\left(x_{1}, \ldots, x_{m}\right)=c\left(x_{1}-\xi_{1}\right) \cdots\left(x_{n}-\xi_{n}\right)
$$

is multiaffine and stable, and $F_{0}(x, \ldots, x)=f(x)$. Let $\Sigma=\left(\left\{i_{k}, j_{k}\right\}: k \in \mathbb{N}\right)$ be a sequence of two-element subsets of $[m]$, and for each $k \in \mathbb{N}$, let $T_{k}=T_{i_{k} j_{k}}^{(1 / 2)}$ and define $F_{k+1}=T_{k}\left(F_{k}\right)$. By induction using Lemma 4.2 , each $F_{k} \in \mathfrak{S}[\mathbf{x}]^{\mathrm{MA}}$ is multiaffine and stable, and $F_{k}(x, \ldots, x)=f(x)$ for all $k \in \mathbb{N}$. We will construct such a sequence $\Sigma$ for which $\left(F_{k}: k \in \mathbb{N}\right)$ converges to $\operatorname{Pol}_{m} f$.

Let $P \in \mathbb{C}[\mathbf{x}]^{\mathrm{MA}}$ be multiaffine, say $P(\mathbf{x})=\sum_{S \subseteq[m]} c(S) \mathbf{x}^{S}$. For $\{i, j\} \subseteq[m]$, let

$$
\omega_{i j}(P)=\sum_{S \subseteq[m]}\left|c(S)-c\left(\tau_{i j}(S)\right)\right|
$$

be the $i j$ th imbalance of $P$, and let $|P|=\sum_{\{i, j\} \subseteq[m]} \omega_{i j}(P)$ be the total imbalance of $P$.

Exercise 4.4. (a) Let $\left(P_{k}: k \in \mathbb{N}\right)$ be polynomials in $\mathbb{C}[\mathbf{x}]^{\mathrm{MA}}$ for which there is a $p \in \mathbb{C}[x]$ such that $P_{k}(x, \ldots, x)=p(x)$ for all $k \in \mathbb{N}$. If $\left|P_{k}\right| \rightarrow 0$ as $k \rightarrow 0$, then $\left(P_{k}: k \in \mathbb{N}\right)$ converges to a limit $P \in \mathbb{C}[\mathbf{x}]^{\mathrm{MA}}$, and $|P|=0$.

(b) For $P \in \mathbb{C}[\mathbf{x}]^{\mathrm{MA}},|P|=0$ if and only if $P$ is invariant under all permutations of $[m]$. Thus, in part (a) the limit is $P=\operatorname{Pol}_{m} p$.

Exercise 4.5. Let $P \in \mathbb{C}[\mathbf{x}]^{\mathrm{MA}}$, let $\{i, j\} \subseteq[m]$, and let $Q=T_{i j}^{(1 / 2)} P$.

(a) Then $\omega_{i j}(Q)=0$.

(b) If $h \in[m] \backslash\{i, j\}$, then $\omega_{h i}(Q) \leq\left(\omega_{h i}(P)+\omega_{h j}(P)\right) / 2$, and similarly for $\omega_{h j}(Q)$.

(c) If $\{h, k\} \subseteq[m] \backslash\{i, j\}$, then $\omega_{h k}(Q)=\omega_{h k}(P)$.

(d) Consequently, $|Q| \leq|P|-\omega_{i j}(P)$.

Now we choose the sequence $\Sigma=\left(\left\{i_{k}, j_{k}\right\}: k \in \mathbb{N}\right)$ as follows: for each $k \in \mathbb{N}$, $\left\{i_{k}, j_{k}\right\} \subseteq[m]$ is any pair of indices $\{i, j\}$ for which $\omega_{i j}\left(F_{k}\right)$ attains its maximum value. Then $\omega_{i_{k} j_{k}}\left(F_{k}\right) \geq\left(\begin{array}{c}m \\ 2\end{array}\right)^{-1}\left|F_{k}\right|$, so that by Exercise $4.5(\mathrm{~d})$ and induction on $k \in \mathbb{N}$,

$$
\left|F_{k+1}\right| \leq\left(1-\left(\begin{array}{c}
m \\
2
\end{array}\right)^{-1}\right)\left|F_{k}\right| \leq\left(1-\left(\begin{array}{c}
m \\
2
\end{array}\right)^{-1}\right)^{k+1}\left|F_{0}\right| .
$$

Thus, by Exercise $4.4, F_{k}$ converges to $\operatorname{Pol}_{m} f$, the $m$ th polarization of $f$. Finally, since each $F_{k}$ is stable (and the limit is a polynomial), Hurwitz's Theorem implies that $\operatorname{Pol}_{m} f$ is stable. This completes the proof of Lemma 4.1, and hence of the Grace-Walsh-Szegő Theorem.

4.4. Polarization arguments. For $\kappa \in \mathbb{N}^{m}$ and a set $\mathcal{S} \subseteq \mathbb{C}[\mathbf{x}]$ of polynomials, let $\mathcal{S}^{\leq \kappa}$ be the set of all $f \in \mathcal{S}$ such that $\operatorname{deg}_{i}(f) \leq \kappa(i)$ for all $i \in[m]$. Let

$$
I(\kappa)=\{(i, j): i \in[m] \text { and } j \in[\kappa(i)]\},
$$

and let $\mathbf{u}=\left\{u_{i j}:(i, j) \in I(\kappa)\right\}$ be indeterminates. For $f \in \mathbb{C}[\mathbf{x}]^{\leq \kappa}$, let $\operatorname{Pol}_{\kappa(i)}^{(i)} f$ denote the $\kappa(i)$ th polarization of $x_{i}$ in $f$ : this is the image of $f$ under the linear 
transformation $\operatorname{Pol}_{\kappa(i)}^{(i)}$ defined by $x_{i}^{j} \mapsto\left(\begin{array}{c}\kappa(i) \\ j\end{array}\right)^{-1} e_{j}\left(u_{i 1}, \ldots, u_{i \kappa(i)}\right)$ for each $j \in[\kappa(i)]$, and linear extension. Finally, the $\kappa$ th polarization of $f$ is

$$
\mathrm{Pol}_{\kappa} f=\mathrm{Pol}_{\kappa(m)}^{(m)} \cdots \mathrm{Pol}_{\kappa(1)}^{(1)} f .
$$

This defines a linear transformation $\mathrm{Pol}_{\kappa}: \mathbb{C}[\mathbf{x}]^{\leq \kappa} \rightarrow \mathbb{C}[\mathbf{u}]^{\mathrm{MA}}$.

Proposition 4.6. Let $\kappa \in \mathbb{N}^{m}$ and $f \in \mathbb{C}[\mathbf{x}]^{\leq \kappa}$. Then $\operatorname{Pol}_{\kappa} f$ is stable if and only if $f$ is stable.

Proof. Diagonalization implies that if $\operatorname{Pol}_{\kappa} f$ is stable, then $f$ is stable, so only the converse implication needs proof. Assume that $f$ is stable, and let $z_{i j} \in \mathcal{H}$ for $(i, j) \in I(\kappa)$. By induction on $m$, repeated application of GWS shows that there are $\mathbf{z}=\left(z_{1}, \ldots, z_{m}\right) \in \mathcal{H}^{m}$ such that

$$
\operatorname{Pol}_{\kappa} f\left(z_{i j}:(i, j) \in I(\kappa)\right)=f(\mathbf{z}) .
$$

Since $f$ is stable, it follows that $\operatorname{Pol}_{\kappa} f$ is stable.

If $f \in \mathbb{R}[\mathbf{x}]^{\leq \kappa}$, then Theorem 3.1 applies to $\operatorname{Pol}_{\kappa} f$. Thus, Proposition 4.6 bootstraps the real stability criterion from multiaffine to arbitrary polynomials. This is a typical application of the GWS Theorem; another is Theorem 5.1 below.

\section{The Borcea-Brändén Master Theorem}

The purpose of this section is to remove the multiaffine condition from the hypothesis of Theorem 3.4.

5.1. Linear transformations preserving stability: polynomial case. The first step is a simple polarization argument.

Theorem 5.1 (Theorem 1.1 of $\left[\underline{5}\right.$ ). Let $\kappa \in \mathbb{N}^{m}$, and let $T: \mathbb{C}[\mathbf{x}]^{\leq \kappa} \rightarrow \mathbb{C}[\mathbf{x}]$ be a linear transformation. Then $T$ maps $\mathfrak{S}[\mathbf{x}]^{\leq \kappa}$ into $\mathfrak{S}[\mathbf{x}]$ if and only if either

(a) $T(f)=\eta(f) \cdot p$ for some linear functional $\eta: \mathbb{C}[\mathbf{x}] \leq \kappa \rightarrow \mathbb{C}$ and $p \in \mathfrak{S}[\mathbf{x}]$, or

(b) the polynomial $T\left((\mathbf{x}+\mathbf{y})^{\kappa}\right)$ is stable in $\mathfrak{S}[\mathbf{x}, \mathbf{y}]$.

Proof. Let $\mathbf{u}=\left\{u_{i j}:(i, j) \in I(\kappa)\right\}$, and define a linear transformation $\widetilde{T}$ : $\mathbb{C}[\mathbf{u}]^{\mathrm{MA}} \rightarrow \mathbb{C}[\mathbf{x}]$ as follows. For every $A \subseteq I(\kappa)$, define $\alpha(A):[m] \rightarrow \mathbb{N}$ by putting $\alpha(A, i)=|\{j \in[\kappa(i)]:(i, j) \in A\}|$ for each $i \in[m]$. Then for each $A \subseteq I(\kappa)$ define $\widetilde{T}\left(\mathbf{u}^{A}\right)=T\left(\mathbf{x}^{\alpha(A)}\right)$, and extend this linearly to all of $\mathbb{C}[\mathbf{u}]^{\mathrm{MA}}$. Let $\Delta: \mathbb{C}[\mathbf{u}]^{\mathrm{MA}} \rightarrow \mathbb{C}[\mathbf{x}]$ be the diagonalization operator defined by $\Delta\left(u_{i j}\right)=x_{i}$ for all $(i, j) \in I(\kappa)$, extended algebraically.

Notice that $T=\widetilde{T} \circ \mathrm{Pol}_{\kappa}$, and that $\widetilde{T}=T \circ \Delta$. By Proposition 4.6 (and Lemma 2.4 ), it follows that $T$ preserves stability if and only if $\widetilde{T}$ preserves stability. This is equivalent to one of two cases in Theorem 3.4.

In case (a), if $\widetilde{T}=p \cdot \widetilde{\eta}$ for some $p \in \mathfrak{S}[\mathbf{x}]$ and linear functional $\widetilde{\eta}: \mathbb{C}[\mathbf{y}]^{\mathrm{MA}} \rightarrow \mathbb{C}$, then $T=p \cdot\left(\eta \circ \mathrm{Pol}_{\kappa}\right)$ is also in case (a). Conversely, if $T$ is in case (a), then the same is true of $\widetilde{T}$, by construction.

In case (b), let $\mathrm{Pol}_{\kappa}^{(\mathbf{y})}: \mathbb{C}[\mathbf{y}]^{\leq \kappa} \rightarrow \mathbb{C}[\mathbf{v}]^{\mathrm{MA}}$ denote the $\kappa$ th polarization of the $\mathbf{y}$ variables. The symbols of $T$ and $\widetilde{T}$ are related by

$$
\widetilde{T}\left((\mathbf{u}+\mathbf{v})^{I(\kappa)}\right)=(T \circ \Delta)\left((\mathbf{u}+\mathbf{v})^{I(\kappa)}\right)=\operatorname{Pol}_{\kappa}^{(\mathbf{y})} T\left((\mathbf{x}+\mathbf{y})^{\kappa}\right),
$$

and Proposition 4.6 shows that $T$ is in case (b) if and only if $\widetilde{T}$ is in case (b). 


\subsection{Linear transformations preserving stability: transcendental case.}

Exercise 5.2. Let $T: \mathbb{C}[\mathbf{x}] \rightarrow \mathbb{C}[\mathbf{x}]$ be a linear transformation.

(a) Then $T: \mathfrak{S}[\mathbf{x}] \rightarrow \mathfrak{S}[\mathbf{x}]$ if and only if $T: \mathfrak{S}[\mathbf{x}]^{\leq \kappa} \rightarrow \mathfrak{S}[\mathbf{x}]$ for all $\kappa \in \mathbb{N}^{m}$

(b) Define $S: \mathbb{C}[\mathbf{x}, \mathbf{y}] \rightarrow \mathbb{C}[\mathbf{x}, \mathbf{y}]$ by $S\left(\mathbf{x}^{\alpha} \mathbf{y}^{\beta}\right)=T\left(\mathbf{x}^{\alpha}\right) \mathbf{y}^{\beta}$ and linear extension. If $T\left((\mathbf{x}+\mathbf{u})^{\kappa}\right)$ is stable for all $\kappa \in \mathbb{N}^{m}$, then $S\left((\mathbf{x}+\mathbf{u})^{\kappa}(\mathbf{y}+\mathbf{v})^{\beta}\right)$ is stable for all $\kappa, \beta \in \mathbb{N}^{m}$.

Let $\overline{\mathfrak{S}[\mathbf{x}]}$ denote the set of all power series in $\mathbb{C}[[\mathbf{x}]]$ that are obtained as the limit of a sequence of stable polynomials in $\mathfrak{S}[\mathbf{x}]$ which converges uniformly on compact sets. Theorem 5.3 is an astounding generalization of the Pólya-Schur Theorem.

Theorem 5.3 (Theorem 1.3 of $[5$, the Borcea-Brändén Master Theorem). Let $T: \mathbb{C}[\mathbf{x}] \rightarrow \mathbb{C}[\mathbf{x}]$ be a linear transformation. Then $T$ maps $\mathfrak{S}[\mathbf{x}]$ into $\mathfrak{S}[\mathbf{x}]$ if and only if either

(a) $T(f)=\eta(f) \cdot p$ for some linear functional $\eta: \mathbb{C}[\mathbf{x}] \rightarrow \mathbb{C}$ and $p \in \mathfrak{S}[\mathbf{x}]$, or

(b) the power series

$$
T\left(\mathrm{e}^{-\mathbf{x y}}\right)=\sum_{\alpha \in \mathbb{N} m}(-1)^{|\alpha|} T\left(\mathbf{x}^{\alpha}\right) \frac{\mathbf{y}^{\alpha}}{\alpha !}
$$

is in $\overline{\mathfrak{S}[\mathbf{x}, \mathbf{y}]}$

(Theorem 3.5 has a similar extension; see Theorems 1.2 and 1.4 of [5].)

Theorem 5.4 (Theorem 5.1 of [5]). Let $F(\mathbf{x}, \mathbf{y})=\sum_{\alpha \in \mathbb{N} m} P_{\alpha}(\mathbf{x}) \mathbf{y}^{\alpha}$ be a power series in $\mathbb{C}[\mathbf{x}][[\mathbf{y}]]$ (so that each $P_{\alpha} \in \mathbb{C}[\mathbf{x}]$ ). Then $F(\mathbf{x}, \mathbf{y})$ is in $\overline{\mathfrak{S}[\mathbf{x}, \mathbf{y}]}$ if and only if, for all $\beta \in \mathbb{N}^{m}$,

$$
\sum_{\alpha \leq \beta}(\beta)_{\alpha} P_{\alpha}(\mathbf{x}) \mathbf{y}^{\alpha}
$$

is stable in $\mathfrak{S}[\mathbf{x}, \mathbf{y}]$.

(This implies the analogous result for real stability, since $\mathfrak{S}_{\mathbb{R}}[\mathbf{x}]=\mathfrak{S}[\mathbf{x}] \cap \mathbb{R}[\mathbf{x}]$.)

Exercise 5.5. Derive Theorem 5.3 from Theorems 5.1 and 5.4. (Hint: $T\left((\mathbf{x}+\mathbf{y})^{\kappa}\right)$ is stable if and only if $T\left((\mathbf{1}-\mathbf{x y})^{\kappa}\right)$ is stable.)

Lemma 5.6 (Lemma 5.2 of [5]). Fix $\beta \in \mathbb{N}^{m}$. The linear transformation $T: \mathbf{y}^{\alpha} \mapsto$ $(\beta)_{\alpha} \mathbf{y}^{\alpha}$ on $\mathbb{C}[\mathbf{y}]$ preserves stability.

Proof. By Theorem 5.1 and Exercise 5.2(a), it suffices to show that for all $\kappa \in \mathbb{N}^{m}$, the polynomial $T\left((\mathbf{y}+\mathbf{u})^{\kappa}\right)$ is stable. Now

$$
T\left((\mathbf{y}+\mathbf{u})^{\kappa}\right)=\prod_{i=1}^{m}\left[\sum_{j=0}^{\kappa(i)} j !\left(\begin{array}{c}
\kappa(i) \\
j
\end{array}\right)\left(\begin{array}{c}
\beta(i) \\
j
\end{array}\right) y_{i}^{j} u_{i}^{\kappa(i)-j}\right],
$$

so it suffices to show that for all $k, b \in \mathbb{N}$, the polynomial $f(t)=\sum_{j=0}^{k} j !\left(\begin{array}{c}k \\ j\end{array}\right)\left(\begin{array}{l}b \\ j\end{array}\right) t^{j}$ is real stable. Let $g(t)=(1+d / d t)^{k} t^{b}$. One can check that $f(t)=t^{b} g(1 / t)$. It thus suffices to show that $1+d / d t$ preserves stability. For any $a \in \mathbb{N},(1+d / d t)(t+u)^{a}=$ $(t+u+a)(t+u)^{a-1}$ is stable, and so Theorem 5.1 implies the result.

Proof of Theorem 5.4. Let $F=F(\mathbf{x}, \mathbf{y})$ be as in the statement of Theorem 5.4, and first assume that $F \in \overline{\mathfrak{S}[\mathbf{x}, \mathbf{y}]}$. Let $\left(F_{n}: n \in \mathbb{N}\right)$ be a sequence of stable polynomials $F_{n}(\mathbf{x}, \mathbf{y})=\sum_{\alpha \in \mathbb{N}^{m}} P_{n, \alpha}(\mathbf{x}) \mathbf{y}^{\alpha}$ in $\mathfrak{S}[\mathbf{x}, \mathbf{y}]$ converging to $F$ uniformly on 
compact sets. Fix $\beta \in \mathbb{N}$ and define a linear transformation $T_{\beta}: \mathbb{C}[\mathbf{x}, \mathbf{y}] \rightarrow \mathbb{C}[\mathbf{x}, \mathbf{y}]$ by $T_{\beta}\left(\mathbf{x}^{\gamma} \mathbf{y}^{\alpha}\right)=(\beta)_{\alpha} \mathbf{x}^{\gamma} \mathbf{y}^{\alpha}$ and linear extension. By Lemma 5.6 and Exercise 5.2, $T_{\beta}$ preserves stability in $\mathfrak{S}[\mathbf{x}, \mathbf{y}]$. Thus, $\left(T_{\beta}\left(F_{n}\right): n \in \mathbb{N}\right)$ is a sequence of stable polynomials converging to $T_{\beta}(F)$. Since $T_{\beta}(F)$ is a polynomial the convergence is uniform on compact sets, and so Hurwitz's Theorem implies that $T_{\beta}(F)$ is stable.

The converse direction is considerably more technical, although the idea is simple. With $F$ as in the theorem, assume that $T_{\beta}(F)$ is stable for all $\beta \in \mathbb{N}^{m}$. For each $n \geq 1$, let

$$
F_{n}(\mathbf{x}, \mathbf{y})=\sum_{\alpha \leq n \mathbf{1}}(n \mathbf{1})_{\alpha} P_{\alpha}(\mathbf{x}) \frac{\mathbf{y}^{\alpha}}{n^{|\alpha|}} .
$$

The sequence $\left(F_{n}: n \geq 1\right)$ converges to $F$, since for each $\alpha \in \mathbb{N}^{m}, n^{-|\alpha|}(n \mathbf{1})_{\alpha} \rightarrow 1$ as $n \rightarrow \infty$. Each $F_{n}$ is stable, by hypothesis (and scaling). Some hard work is required to show that $\left(F_{n}\right)$ converges uniformly on compact sets. This appears as Lemma 5.11 below.

\subsection{A multivariate Szász principle.}

Lemma 5.7 (Szász, Lemma 5.3 of [5]). Assume that

$$
f(x)=\sum_{i=0}^{d} c_{i} x^{i}=\prod_{j=1}^{d}\left(1+a_{j} x\right)
$$

is stable (note that $\left.c_{0}=1\right)$. Then

$$
\sum_{j=1}^{d}\left|a_{j}\right|^{2} \leq 3\left|c_{1}\right|^{2}+2\left|c_{2}\right|
$$

Proof. Since $f$ is stable, $\operatorname{Im}\left(a_{j}\right) \leq 0$ for all $j \in[d]$, so that

$$
\sum_{j=1}^{d} \operatorname{Im}\left(a_{j}\right)^{2} \leq\left(\sum_{j=1}^{d} \operatorname{Im}\left(a_{j}\right)\right)^{2}=\operatorname{Im}\left(c_{1}\right)^{2} .
$$

Note that $\sum_{j=1}^{d} a_{j}^{2}=c_{1}^{2}-2 c_{2}$, and that for any $z \in \mathbb{C}$,

$$
|z|^{2}=\left(\operatorname{Re}(z)^{2}-\operatorname{Im}(z)^{2}\right)+2 \operatorname{Im}(z)^{2}=\operatorname{Re}\left(z^{2}\right)+2 \operatorname{Im}(z)^{2} .
$$

It follows that

$$
\begin{aligned}
\sum_{j=1}^{d}\left|a_{j}\right|^{2} & =\sum_{j=1}^{d}\left(\operatorname{Re}\left(a_{j}^{2}\right)+2 \operatorname{Im}\left(a_{j}\right)^{2}\right) \\
& \leq \operatorname{Re}\left(c_{1}^{2}-2 c_{2}\right)+2 \operatorname{Im}\left(c_{1}\right)^{2} \leq 3\left|c_{1}\right|^{2}+2\left|c_{2}\right| .
\end{aligned}
$$

Lemma 5.8 (Lemma 5.4 of [5]). Assume that $f(\mathbf{x})=\sum_{\alpha \in \mathbb{N}_{m}} c(\alpha) \mathbf{x}^{\alpha}$ is a stable polynomial with $c(\mathbf{0})=1$, and let

$$
A=\left(3\left(\sum_{i=1}^{m}\left|c\left(\delta_{i}\right)\right|\right)^{2}+2 \sum_{i, j=1}^{m}\left|c\left(\delta_{i}+\delta_{j}\right)\right|\right)^{1 / 2} .
$$

Then, for all $\beta \in \mathbb{N}^{m}$,

$$
|c(\beta)| \leq|\beta|^{-|\beta| / 2} \frac{\beta^{\beta}}{\beta !} A^{|\beta|} .
$$


Proof. Consider any $\beta \in \mathbb{N}^{m}$, and let $d=|\beta|$. If $c(\beta)=0$, then there is nothing to prove, so assume that $c(\beta) \neq 0$. The linear transformation $\mathbf{x}^{\alpha} \mapsto(\beta)_{\alpha} \beta^{-\alpha} \mathbf{x}^{\alpha}$ preserves stability, by Lemma 5.6 (and scaling). Let $g(x)$ be the univariate polynomial obtained by applying this transformation to $f(\mathbf{x})$ and then fully diagonalizing the indeterminates (setting all $\left.x_{i}=x\right)$. Then $g(x)=\sum_{i=0}^{d} c_{i} x^{i}=\prod_{j=1}^{d}\left(1+a_{j} x\right.$ ) is stable, of degree $d$, with $c_{d}=\beta ! \beta^{-\beta} c(\beta)$. From Lemma 5.7 it follows that

$$
\left|\frac{\beta !}{\beta^{\beta}} c(\beta)\right|=\prod_{j=1}^{d}\left|a_{j}\right| \leq\left(\frac{1}{d} \sum_{j=1}^{d}\left|a_{j}\right|^{2}\right)^{d / 2} \leq\left(3\left|c_{1}\right|^{2}+2\left|c_{2}\right|\right)^{d / 2} d^{-d / 2} \leq d^{-d / 2} A^{d},
$$

as desired.

For a polynomial $f \in \mathbb{C}[\mathbf{x}]$, let $|f|_{r}=\max \left\{|f(\mathbf{z})|:\left|z_{i}\right| \leq r\right.$ for all $\left.i \in[m]\right\}$.

Proposition 5.9 (Theorem 5.5 of [5]). Assume that $f(\mathbf{x})=\sum_{\alpha \in \mathbb{N}^{m}} c(\alpha) \mathbf{x}^{\alpha}$ is a stable polynomial with $c(\mathbf{0})=1$, let $A$ be as in Lemma 5.8, let

$$
B=2^{m-1} \frac{\sqrt{2 \mathrm{e}^{2}-\mathrm{e}}}{\mathrm{e}-1},
$$

and let $C=2 \mathrm{e}^{2} A^{2}$. Then for all $r \geq 0,|f|_{r} \leq B \mathrm{e}^{C r^{2}}$.

Proof. We use the following elementary inequalities: for all $m \in \mathbb{N}$,

$$
\mathrm{e}^{-m} \leq \frac{m !}{m^{m}} \leq(m \mathrm{e}+1) \mathrm{e}^{-m} .
$$

For $k \in \mathbb{N}$, let $s(k)=\sum_{\beta \in \mathbb{N}^{m}:|\beta|=k} \beta^{\beta} / \beta$ !. From the above inequalities, we obtain the (rough) estimate

$$
s(k) \leq\left(\begin{array}{c}
m-1+k \\
k
\end{array}\right) \mathrm{e}^{k} \leq 2^{m-1+k} \mathrm{e}^{k} .
$$

Applying Lemma 5.8, we find that

$$
\begin{aligned}
|f|_{r} & \leq \sum_{\beta \in \mathbb{N}^{m}}|\beta|^{-|\beta| / 2} \frac{\beta^{\beta}}{\beta !}(A r)^{|\beta|} \\
& =\sum_{k=0}^{\infty} s(k) k^{-k / 2}(A r)^{k} \leq 2^{m-1} \sum_{k=0}^{\infty} k^{-k / 2}(2 \mathrm{e} A r)^{k} .
\end{aligned}
$$

Let $R=2 \mathrm{e} A r$. By the Cauchy-Schwartz inequality (and the elementary inequalities above), it follows that

$$
\begin{aligned}
\sum_{k=0}^{\infty} k^{-k / 2}(2 \mathrm{e} A r)^{k} & \leq \sum_{k=0}^{\infty}\left((k \mathrm{e}+1) \mathrm{e}^{-k}\right)^{1 / 2}\left(\frac{R^{2 k}}{k !}\right)^{1 / 2} \\
& \leq\left(\sum_{k=0}^{\infty}(k \mathrm{e}+1) \mathrm{e}^{-k}\right)^{1 / 2}\left(\sum_{k=0}^{\infty} \frac{R^{2 k}}{k !}\right)^{1 / 2}=\frac{\sqrt{2 \mathrm{e}^{2}-\mathrm{e}}}{\mathrm{e}-1} \cdot \mathrm{e}^{R^{2} / 2} .
\end{aligned}
$$

This yields the result.

Here is the multivariate Szász Principle of Borcea and Brändén. 
Theorem 5.10 (Theorem 5.6 of [5]). Assume that $f(\mathbf{x})=\sum_{\alpha \in \mathbb{N}^{m}} c(\alpha) \mathbf{x}^{\alpha}$ is a stable polynomial, let $\mathcal{M}(f)$ be the set of minimal elements $\beta \in \mathbb{N}^{m}$ for which $c(\beta) \neq 0$, and let $\mathcal{M}_{2}(f)=\{\beta+\gamma: \beta \in \mathcal{M}(f)$ and $|\gamma| \leq 2\}$. Then there are constants $B$ and $C$, depending only on the coefficients $c(\alpha)$ with $\alpha \in \mathcal{M}_{2}(f)$, such that for all $r \geq 0,|f|_{r} \leq B \mathrm{e}^{C r^{2}}$. Moreover, $B$ and $C$ can be taken as continuous nondecreasing functions of $\left\{|c(\alpha)|: \alpha \in \mathcal{M}_{2}(f)\right\}$.

Proof. Let $k=\max \{|\alpha|: \alpha \in \mathcal{M}(f)\}$. We proceed by induction on $m+k$. If $f(\mathbf{0}) \neq 0$, then $k=0$, and the existence of $B$ and $C$ follows from Proposition 5.9. If $m=1$, then $f(x)=x^{a} g(x)$ with $a \in \mathbb{N}$ and $g(0) \neq 0$. Applying Proposition 5.9 to $g(x)$ (together with $r \leq \mathrm{e}^{r^{2}}$ ) yields the result. These cases form the basis of induction.

Let $\alpha \in \mathcal{M}(f)$ be such that $|\alpha|=k \leq 1$, and (by permutation of indeterminates) assume that $\alpha(1) \geq 1$. Since

$$
f\left(x_{1}, x_{2}, \ldots, x_{m}\right)=f\left(0, x_{2}, \ldots, x_{m}\right)+x_{1} \int_{0}^{1} \partial_{1} f\left(x_{1} t, x_{2}, \ldots, x_{m}\right) d t
$$

it follows that $|f|_{r} \leq|g|_{r}+r|h|_{r}$, in which $g=\left.f\right|_{x_{1}=0}$ and $h=\partial_{1} f$. By induction there are constants $B_{1}, B_{2}, C_{1}$, and $C_{2}$ such that $|g|_{r} \leq B_{1} \mathrm{e}^{C_{1} r^{2}}$ and $|h|_{r} \leq B_{2} \mathrm{e}^{C_{2} r^{2}}$. Taking $B=2 \max \left\{B_{1}, B_{2}\right\}$ and $C=\max \left\{C_{1}, C_{2}+1\right\}$, we see that $|f|_{r} \leq B \mathrm{e}^{C r^{2}}$, completing the induction step and the proof. (The dependence of $B$ and $C$ on only the coefficients $c(\alpha)$ with $\alpha \in \mathcal{M}_{2}(f)$ carries through the induction step easily, as does continuity and the nondecreasing property.)

Lemma 5.11. Returning to the proof of Theorem 5.4, the sequence $\left(F_{n}: n \geq 1\right)$ converges uniformly on compact sets. Hence, $F(\mathbf{x}, \mathbf{y})$ is in $\overline{\mathfrak{S}[\mathbf{x}, \mathbf{y}]}$.

Proof. Let $\mathcal{M}(F)$ be the set of minimal elements $\gamma \in \mathbb{N}^{2 m}$ such that the monomial $x_{1}^{\gamma(1)} \cdots x_{m}^{\gamma(m)} y_{1}^{\gamma(m+1)} \cdots y_{m}^{\gamma(2 m)}$ occurs in $F(\mathbf{x}, \mathbf{y})$ with a nonzero coefficient, and let $\mathcal{M}_{2}(F)=\{\gamma+\kappa: \gamma \in \mathcal{M}(F)$ and $|\kappa| \leq 2\}$. Then $\mathcal{M}(F)$ is a subset of $\mathbb{N}^{2 m}$ in which no two elements are comparable, and it follows that $\mathcal{M}(F)$ is finite. Thus $\mathcal{M}_{2}(F)$ is also finite. Let $N$ be the maximum among all coordinates of all $\gamma \in \mathcal{M}_{2}(F)$. From the definition of $F_{n}$ it follows that $\mathcal{M}_{2}\left(F_{n}\right)=\mathcal{M}_{2}(F)$ for all $n \geq N$. Let

$$
F(\mathbf{x}, \mathbf{y})=\sum_{\gamma \in \mathbb{N}^{2 m}} c(\gamma) x_{1}^{\gamma(1)} \cdots x_{m}^{\gamma(m)} y_{1}^{\gamma(m+1)} \cdots y_{m}^{\gamma(2 m)},
$$

and for $n \geq 1$ let

$$
F_{n}(\mathbf{x}, \mathbf{y})=\sum_{\gamma \in \mathbb{N}^{2 m}} c_{n}(\gamma) x_{1}^{\gamma(1)} \cdots x_{m}^{\gamma(m)} y_{1}^{\gamma(m+1)} \cdots y_{m}^{\gamma(2 m)} .
$$

From the definition of $F_{n}$ it follows that for all $n \geq 1$ and $\gamma \in \mathbb{N}^{2 m},\left|c_{n}(\gamma)\right| \leq|c(\gamma)|$.

Each $F_{n}$ is a stable polynomial, so let $B_{n}$ and $C_{n}$ be the constants as in Theorem 5.10 , so that $\left|F_{n}\right|_{r} \leq B_{n} \mathrm{e}^{C_{n} r^{2}}$ for all $r>0$ and all $n \geq 1$. Let $B^{\prime}$ and $C^{\prime}$ be the constants determined in Theorem 5.10 from the data $\left\{|c(\gamma)|: \gamma \in \mathcal{M}_{2}(F)\right\}$. Since $\mathcal{M}_{2}\left(F_{n}\right)=\mathcal{M}_{2}(F)$ is constant for $n \geq N$, and $\left|c_{n}(\gamma)\right| \leq|c(\gamma)|$ for all $n \geq N$ and $\gamma \in \mathcal{M}_{2}(F)$, from Theorem 5.10 it follows that $B_{n} \leq B^{\prime}$ and $C_{n} \leq C^{\prime}$ for all $n \geq N$. Therefore,

$$
B=\sup \left\{B_{n}: n \geq 1\right\}=\max \left\{B_{1}, B_{2}, \ldots, B_{N-1}, B^{\prime}\right\}
$$

and

$$
C=\sup \left\{C_{n}: n \geq 1\right\}=\max \left\{C_{1}, C_{2}, \ldots, C_{N-1}, C^{\prime}\right\}
$$


are finite. Thus $\left|F_{n}\right|_{r} \leq B \mathrm{e}^{C r^{2}}$ for all $n \geq 1$. This bound depends on $r$ but not on $n$-that is, $\left(F_{n}: n \geq 1\right)$ is a locally bounded sequence.

By Vitali's Theorem, every locally bounded convergent sequence of holomorphic functions converges uniformly on compact sets to a holomorphic function; see Exercise 1.4.37 of Scheidemann [17, for example. Applying this fact to the sequence $\left(F_{n}: n \geq 1\right)$ yields the desired result.

\section{JoHNSON'S CONJECTURES}

Let $\mathcal{A}=\left(A_{1}, \ldots, A_{k}\right)$ be a $k$-tuple of $n$-by- $n$ matrices. Define the mixed determinant of $\mathcal{A}$ to be

$$
\operatorname{Det}(\mathcal{A})=\operatorname{Det}\left(A_{1}, \ldots, A_{k}\right)=\sum_{\left(S_{1}, \ldots, S_{k}\right)} \prod_{i=1}^{k} \operatorname{det} A_{i}\left[S_{i}\right],
$$

in which the sum is over all ordered sequences of $k$ pairwise disjoint subsets of $[n]$ such that $[n]=S_{1} \cup \cdots \cup S_{k}$, and $A_{i}\left[S_{i}\right]$ is the principal submatrix of $A_{i}$ supported on rows and columns in $S_{i}$. Let $A_{i}\left(S_{i}\right)$ be the complementary principal submatrix supported on rows and columns not in $S_{i}$, and for $j \in[n]$, let $A_{i}(j)=A_{i}(\{j\})$.

For example, when $k=2$ and $A_{1}=x I$ and $A_{2}=-B$, this specializes to $\operatorname{Det}(x I,-B)=\operatorname{det}(x I-B)$, the characteristic polynomial of $B$. In the late 1980s, Johnson made three conjectures about the $k=2$ case more generally.

Johnson's Conjectures. Let $A$ and $B$ be n-by-n matrices, with $A$ positive definite and $B$ Hermitian.

(a) Then $\operatorname{Det}(x A,-B)$ has only real roots.

(b) For $j \in[n]$, the roots of $\operatorname{Det}(x A(j),-B(j))$ interlace those of $\operatorname{Det}(x A,-B)$.

(c) The inertia of $\operatorname{Det}(x A,-B)$ is the same as that of $\operatorname{det}(x I-B)$.

In part (c), the inertia of a univariate real stable polynomial $p$ is the triple $\iota(p)=\left(\iota_{-}(p), \iota_{0}(p), \iota_{+}(p)\right)$ with entries the number of negative, zero, or positive roots of $p$, respectively.

In 2008, Borcea and Brändén 1] proved all three of these statements in much greater generality.

Theorem 6.1 (Theorem 2.6 of [1]). Fix integers $\ell, m, n \geq 1$. For $h \in[\ell]$ and $i \in[m]$, let $B_{h}$ and $A_{h i}$ be $n$-by-n matrices, and let

$$
L_{h}=\sum_{i=1}^{m} x_{i} A_{h i}+B_{h}
$$

(a) If all the $A_{h i}$ are positive semidefinite and all the $B_{h}$ are Hermitian, then $\operatorname{Det}(\mathcal{L})=\operatorname{Det}\left(L_{1}, \ldots, L_{\ell}\right) \in \mathfrak{S}_{\mathbb{R}}[\mathbf{x}]$ is real stable.

(b) For each $j \in[n]$, let $\mathcal{L}(j)=\left(L_{1}(j), \ldots, L_{\ell}(j)\right)$. With the hypotheses of part (a), the polynomial $\operatorname{Det}(\mathcal{L})+y \operatorname{Det}(\mathcal{L}(j)) \in \mathfrak{S}_{\mathbb{R}}[\mathbf{x}, y]$ is real stable.

Proof. Let $Y=\operatorname{diag}\left(y_{1}, \ldots, y_{n}\right)$ be a diagonal matrix of indeterminates. By Proposition 2.1 , for each $h \in[\ell]$ the polynomial

$$
\operatorname{det}\left(Y+L_{h}\right)=\sum_{S \subseteq[n]} \mathbf{y}^{S} \operatorname{det} L_{h}(S)
$$


is real stable in $\mathfrak{S}_{\mathbb{R}}[\mathbf{x}, \mathbf{y}]$. By inversion of all the $\mathbf{y}$ indeterminates, each

$$
\operatorname{det}\left(I-Y L_{h}\right)=\sum_{S \subseteq[n]}(-1)^{|S|} \mathbf{y}^{S} \operatorname{det} L_{h}[S]
$$

is real stable. Since $\prod_{h=1}^{\ell} \operatorname{det}\left(I-Y L_{h}\right)$ is real stable, contraction and specialization imply that

$$
\operatorname{Det}(\mathcal{L})=\left.(-1)^{n} \frac{\partial^{n}}{\partial y_{1} \cdots \partial y_{n}} \prod_{h=1}^{\ell} \operatorname{det}\left(I-Y L_{h}\right)\right|_{\mathbf{y}=\mathbf{0}}
$$

is real stable, proving part (a).

For part (b), let $V$ be the $n$-by- $n$ matrix with all entries zero except for $V_{j j}=y$. By part (a),

$$
\operatorname{Det}\left(V, L_{1}, \ldots, L_{h}\right)=\operatorname{Det}(\mathcal{L})+y \operatorname{Det}(\mathcal{L}(j))
$$

is real stable.

Theorem 6.1 (with Corollary 2.10) clearly settles conjectures (a) and (b).

Proof of conjecture (c). Let $A$ and $B$ be $n$-by- $n$ matrices with $A$ positive definite and $B$ Hermitian. Let $\left(\iota_{-}, \iota_{0}, \iota_{+}\right)$be the inertia of $\operatorname{det}(x I-B)$. Let $f(x)=$ $\operatorname{Det}(x A,-B)$, and let $\left(\nu_{-}, \nu_{0}, \nu_{+}\right)$be the inertia of $f$.

We begin by showing that $\nu_{0}=\iota_{0}$. Since $\iota_{0}=\min \{|S|: S \subseteq[n]$ and $\operatorname{det}(B(S)) \neq$ $0\}$, it follows that $\nu_{0} \geq \iota_{0}$. The constant term of $f(x)$ is $(-1)^{n} \operatorname{det}(B)$, so that if $\iota_{0}=0$, then $\nu_{0}=0$. If $\iota_{0}=k>0$, then let $S=\left\{s_{1}, \ldots, s_{k}\right\} \subseteq[n]$ be such that $\operatorname{det}(B(S)) \neq 0$. For $0 \leq i \leq k$, let $f_{i}(x)=\operatorname{Det}\left(A\left(\left\{s_{1}, \ldots, s_{i}\right\}\right),-B\left(\left\{s_{1}, \ldots, s_{i}\right\}\right)\right)$, so that $f_{0}(x)=f(x)$. By Theorem 6.1 , the roots of $f_{i-1}$ and of $f_{i}$ are interlaced for each $i \in[k]$. Thus,

$$
\nu_{0}=\iota_{0}\left(f_{0}\right) \leq \iota_{0}\left(f_{1}\right)+1 \leq \iota_{0}\left(f_{2}\right)+2 \leq \cdots \leq \iota_{0}\left(f_{k}\right)+k=k=\iota_{0},
$$

since $\iota_{0}\left(f_{k}\right)=0$ because $\operatorname{det}(B(S)) \neq 0$. Therefore $\nu_{0}=\iota_{0}$.

For any positive definite matrix $A, \operatorname{Det}(x A,-B)$ is a polynomial of degree $n$. Suppose that $A$ is such a matrix for which $\nu_{+} \neq \iota_{+}$. Consider the matrices $A_{\lambda}=$ $(1-\lambda) I+\lambda A$ for $\lambda \in[0,1]$. Each of these matrices is positive definite. From the paragraph above, each of the polynomials $g_{\lambda}(x)=\operatorname{Det}\left(x A_{\lambda},-B\right)$ has $\iota_{0}\left(g_{\lambda}\right)=\iota_{0}$. Since $\iota_{+}\left(g_{0}\right)=\iota_{+} \neq \nu_{+}=\iota_{+}\left(g_{1}\right)$ and the roots of $g_{\lambda}$ vary continuously with $\lambda$, there is some value $\mu \in(0,1)$ for which $\iota_{0}\left(g_{\mu}\right)>\iota_{0}$. This contradiction shows that $\nu_{+}=\iota_{+}$, and hence $\nu_{-}=\iota_{-}$as well.

Borcea and Brändén [1] proceed to derive many inequalities for the principal minors of positive semidefinite matrices, and some for merely Hermitian matrices. These are applications of inequalities valid more generally for real stable polynomials. The simplest of these inequalities are as follows.

For an $n$-by- $n$ matrix $A$, the $j$ th symmetrized Fisher product is

$$
\sigma_{j}(A)=\sum_{S \subseteq[n]:|S|=j} \operatorname{det}(A[S]) \operatorname{det}(A(S)),
$$

and the $j$ th averaged Fisher product is $\widehat{\sigma}_{j}(A)=\left(\begin{array}{c}n \\ j\end{array}\right)^{-1} \sigma_{j}(A)$. Notice that $\sigma_{j}(A)=$ $\sigma_{n-j}(A)$ for all $0 \leq j \leq n$. 
Corollary 6.2. Let $A$ be an n-by-n positive semidefinite matrix.

(a) Then $\widehat{\sigma}_{j}(A)^{2} \geq \widehat{\sigma}_{j-1}(A) \widehat{\sigma}_{j+1}(A)$ for all $1 \leq j \leq n-1$.

(b) Also, $\widehat{\sigma}_{0}(A) \leq \widehat{\sigma}_{1}(A) \leq \cdots \leq \widehat{\sigma}_{\lfloor n / 2\rfloor}$.

(c) If $A$ is positive definite and $\operatorname{det}(A)=d$, then

$$
\frac{\widehat{\sigma}_{1}(A)}{d} \geq\left(\frac{\widehat{\sigma}_{2}(A)}{d}\right)^{1 / 2} \geq\left(\frac{\widehat{\sigma}_{3}(A)}{d}\right)^{1 / 3} \geq \cdots \geq\left(\frac{\widehat{\sigma}_{n}(A)}{d}\right)^{1 / n}=1 .
$$

Proof. It suffices to consider positive definite $A$. By Theorem 6.1, the polynomial $\operatorname{Det}(x A,-A)=\sum_{j=0}^{n}(-1)^{j} \sigma_{j}(A) x^{j}$ has only real roots, and these roots are all positive. Part (a) follows from Newton's Inequalities [14, Theorem 51]. Part (a) and the symmetry $\sigma_{j}(A)=\sigma_{n-j}(A)$ for all $0 \leq j \leq n$ imply part (b). Part (c) follows from Maclaurin's Inequalities [14, Theorem 52].

\section{THE SYMMETRIC EXCLUSION PROCESS}

This section summarizes an application of stable polynomials to probability and statistical mechanics from a 2009 paper of Borcea, Brändén and Liggett [7].

Let $\Lambda$ be a set of sites. A symmetric exclusion process (SEP) is a type of Markov chain with state space a subset of $\{0,1\}^{\Lambda}$. In a state $S: \Lambda \rightarrow\{0,1\}$, the sites in $S^{-1}(1)$ are occupied and the sites in $S^{-1}(0)$ are vacant. This is meant to model a physical system of particles interacting by means of hard-core exclusions. Such models come in many varieties; to avoid technicalities we discuss only the case of a finite system $\Lambda$ and continuous time $t$. (The results of this section extend to countable $\Lambda$ under a reasonable finiteness condition on the interaction rates.) Symmetry of the interactions turns out to be crucial, but particle number conservation is unimportant.

Let $E$ be a set of two-element subsets of $\Lambda$. For each $\{i, j\} \in E$, let $\lambda_{i j}>0$ be a positive real, and let $\tau_{i j}: \Lambda \rightarrow \Lambda$ be the permutation that exchanges $i$ and $j$ and fixes all other sites. Our SEP Markov chain $\mathcal{M}$ proceeds as follows. Each $\{i, j\} \in E$ has a Poisson process clock of rate $\lambda_{i j}$, and these are independent of one another. With probability one, no two clocks ever ring at the same time. When the clock of $\{i, j\}$ rings, the current state $S$ is updated to the new state $S \circ \tau_{i j}$. In other words, when the $\{i, j\}$ clock rings, if exactly one of the sites $\{i, j\}$ is occupied, then a particle hops from the occupied to the vacant of these two sites.

Let $\Lambda=[m]$ and $\Omega=\{0,1\}^{\Lambda}$, let $\varphi_{0}$ be an initial probability distribution on $\Omega$, and let $\varphi_{t}$ be the distribution of the state of $\mathcal{M}$, starting at $\varphi_{0}$, after evolving for time $t \geq 0$. We are concerned with properties of the distribution $\varphi_{t}$ that hold for all $t \geq 0$.

7.1. Negative correlation and negative association. Consider a probability distribution $\varphi$ on $\Omega$. An event $\mathcal{E}$ is any subset of $\Omega$. The probability of the event $\mathcal{E}$ is $\operatorname{Pr}[\mathcal{E}]=\sum_{S \in \mathcal{E}} \varphi(S)$. An event $\mathcal{E}$ is increasing if whenever $S \leq S^{\prime}$ in $\Omega$ and $S \in \mathcal{E}$, then $S^{\prime} \in \mathcal{E}$. For example, if $K$ is any subset of $\Lambda$ and $\mathcal{E}_{K}$ is the event that all sites in $K$ are occupied, then $\mathcal{E}_{K}$ is an increasing event. Notice that this event has the form $\mathcal{E}_{K}=\mathcal{E}^{\prime} \times\{0,1\}^{\Lambda \backslash K}$ for some event $\mathcal{E}^{\prime} \subseteq\{0,1\}^{K}$. Two events $\mathcal{E}$ and $\mathcal{F}$ are disjointly supported when one can partition $\Lambda=A \cup B$ with $A \cap B=\varnothing$ and $\mathcal{E}=\mathcal{E}^{\prime} \times\{0,1\}^{B}$ and $\mathcal{F}=\{0,1\}^{A} \times \mathcal{F}^{\prime}$ for some events $\mathcal{E}^{\prime} \subseteq\{0,1\}^{A}$ and $\mathcal{F}^{\prime} \subseteq\{0,1\}^{B}$.

A probability distribution on $\Omega$ is negatively associated (NA) when $\operatorname{Pr}[\mathcal{E} \cap \mathcal{F}] \leq$ $\operatorname{Pr}[\mathcal{E}] \cdot \operatorname{Pr}[\mathcal{F}]$ for any two increasing events that are disjointly supported. It is 
negatively correlated (NC) when $\operatorname{Pr}\left[\varepsilon_{\{i, j\}}\right] \leq \operatorname{Pr}\left[\varepsilon_{\{i\}}\right] \cdot \operatorname{Pr}\left[\varepsilon_{\{j\}}\right]$ for any two distinct sites $\{i, j\} \subseteq \Lambda$. Clearly NA implies NC.

It is useful to find conditions under which NC implies NA, since NC is so much easier to check. The following originates with Feder and Mihail, but many others have contributed their insights; see Section 4.2 of [7]. The partition function of any $\varphi: \Omega \rightarrow \mathbb{R}$ is the real multiaffine polynomial

$$
Z(\varphi)=Z(\varphi ; \mathbf{x})=\sum_{S \in \Omega} \varphi(S) \mathbf{x}^{S}
$$

in $\mathbb{R}[\mathbf{x}]^{\mathrm{MA}}$. If $\varphi$ is nonzero and nonnegative, then for any $\mathbf{a} \in \mathbb{R}^{\Lambda}$ with $\mathbf{a}>\mathbf{0}$, this defines a probability distribution $\varphi^{\mathbf{a}}: \Omega \rightarrow[0,1]$ by setting $\varphi^{\mathbf{a}}(S)=\varphi(S) \mathbf{a}^{S} / Z(\varphi ; \mathbf{a})$ for all $S \in \Omega$.

Feder-Mihail Theorem (Theorem 4.8 of $[7]$ ). Let $\mathcal{S}$ be a class of nonzero nonnegative functions satisfying the following conditions:

(i) Each $\varphi \in \mathcal{S}$ has domain $\{0,1\}^{\Lambda}$ for some finite set $\Lambda=\Lambda(\varphi)$.

(ii) For each $\varphi \in \mathcal{S}, Z(\varphi)$ is a homogeneous polynomial.

(iii) For each $\varphi \in \mathcal{S}$ and $i \in \Lambda(\varphi),\left.Z(\varphi)\right|_{x_{i}=0}$ and $\partial_{i} Z(\varphi)$ are partition functions of members of $\mathcal{S}$.

(iv) For each $\varphi \in \mathcal{S}$ and $\mathbf{a} \in \mathbb{R}^{\Lambda(\varphi)}$ with $\mathbf{a}>\mathbf{0}, \varphi^{\mathbf{a}}$ is $N C$. Then for every $\varphi \in \mathcal{S}$ and $\mathbf{a} \in \mathbb{R}^{\Lambda(\varphi)}$ with $\mathbf{a}>\mathbf{0}, \varphi^{\mathbf{a}}$ is $N A$.

7.2. A conjecture of Liggett and Pemantle. In the early 2000s, Liggett and Pemantle arrived independently at the following conjecture, now a theorem.

Theorem 7.1 (Theorem 5.2 of [7]). If the initial distribution $\varphi_{0}$ of an SEP is deterministic (i.e., concentrated on a single state), then $\varphi_{t}$ is $N A$ for all $t \geq 0$.

Proof. This amounts to finding a class $\mathfrak{S}$ of probability distributions such that:

(1) deterministic distributions are in $\mathfrak{S}$,

(2) being in $\mathfrak{S}$ implies NA, and

(3) time evolution of the SEP preserves membership in $\mathfrak{S}$.

Borcea, Brändén, and Liggett [7] identified such a class: $\varphi$ is in $\mathfrak{S}$ if and only if the partition function $Z(\varphi)$ is homogeneous, multiaffine, and real stable. (Notice that if $\varphi$ is in $\mathfrak{S}$, then $\varphi^{\mathbf{a}}$ is in $\mathfrak{S}$ for all $\mathbf{a} \in \mathbb{R}^{\Lambda}$ with $\mathbf{a}>\mathbf{0}$, by scaling.) We proceed to check the three claims above.

Claim (1) is trivial, since if $\varphi(S)=1$, then $Z(\varphi)=\mathbf{x}^{S}$, which is clearly homogeneous, multiaffine, and real stable.

To check claim (2) we verify the hypotheses of the Feder-Mihail Theorem. Hypotheses (i) and (ii) hold since $Z(\varphi)$ is multiaffine and homogeneous. By specialization and contraction, (iii) holds. To check (iv), let $\mathbf{a} \in \mathbb{R}^{\Lambda}$ with $\mathbf{a}>\mathbf{0}$, let $\{i, j\} \subseteq \Lambda$, and consider the probability distribution $\varphi^{\mathbf{a}}$ on $\Omega$. The occupation probability for site $i$ is

$$
\operatorname{Pr}\left[\mathcal{E}_{\{i\}}\right]=\sum_{S \in\{0,1\}^{\Lambda}: S(i)=1} \frac{\varphi(S) \mathbf{a}^{S}}{Z(\varphi ; \mathbf{a})}=a_{i} \frac{\partial_{i} Z(\varphi ; \mathbf{a})}{Z(\varphi ; \mathbf{a})},
$$

and similarly for $\operatorname{Pr}\left[\mathcal{E}_{\{j\}}\right]$. Likewise, $\operatorname{Pr}\left[\mathcal{E}_{\{i, j\}}\right]=a_{i} a_{j} Z(\varphi ; \mathbf{a})^{-1} \cdot \partial_{i} \partial_{j} Z(\varphi ; \mathbf{a})$. Now

$$
\operatorname{Pr}\left[\mathcal{E}_{\{i, j\}}\right]-\operatorname{Pr}\left[\mathcal{E}_{\{i\}}\right] \cdot \operatorname{Pr}\left[\mathcal{E}_{\{j\}}\right]=-\frac{a_{i} a_{j}}{Z(\varphi ; \mathbf{a})^{2}} \cdot \Delta_{i j} Z(\varphi ; \mathbf{a}) \leq 0,
$$


by Theorem 3.1. Thus $\varphi^{\mathbf{a}}$ is NC. By the Feder-Mihail Theorem, every $\varphi$ in $\mathfrak{S}$ is NA.

To check claim (3) we need some of the theory of continuous time Markov chains. The time evolution of a Markov chain $\mathcal{M}$ with finite state space $\Omega$ is governed by a one-parameter semigroup $T(t)$ of transformations of $\mathbb{R}^{\Omega}$. For a function $F \in \mathbb{R}^{\Omega}$ and time $t \geq 0$ and state $S \in \Omega,(T(t) F)(S)$ is the expected value of $F$ at time $t$, given that the initial distribution of $\mathcal{M}$ is concentrated at $S$ with probability one at time 0 . In particular, $\varphi_{t}=T(t) \varphi_{0}$ for all $t \geq 0$, and all initial distributions $\varphi_{0}$. In the case of the SEP we are considering, the infinitesimal generator $\mathcal{L}$ of the semigroup $T(t)$ is given by

$$
\mathcal{L}=\sum_{\{i, j\} \in E} \lambda_{i j}\left(\tau_{i j}-1\right)
$$

For each $\{i, j\} \in E$, this replaces each $S \in \Omega$ by $S \circ \tau_{i j}$ at the rate $\lambda_{i j}$.

In preparation for Section 7.3, it is useful to regard $\mathcal{L}$ as an element of the real semigroup algebra $\mathfrak{A}=\mathbb{R}[\mathfrak{E}]$ of the semigroup $\mathfrak{E}$ of all endofunctions $\mathfrak{f}: \Omega \rightarrow \Omega$ (with the operation of functional composition). The left action of $\mathfrak{E}$ on $\Omega$ is extended to a left action of $\mathfrak{A}$ on $\mathbb{C}[\mathbf{x}]$ as usual-for $\mathfrak{f} \in \mathfrak{E}$ and $S \in \Omega, \mathfrak{f}\left(\mathbf{x}^{S}\right)=\mathbf{x}^{\mathfrak{f}(S)}$, extended bilinearly to all of $\mathfrak{A}$ and $\mathbb{C}[\mathbf{x}]$. A permutation $\sigma \in \mathcal{S}(\Lambda)$ is identified with the endofunction $\mathfrak{f}_{\sigma}: S \mapsto S \circ \sigma^{-1}$, so this action of $\mathfrak{A}$ agrees with the action of $\mathcal{S}(m)$ in Section 4.2. A left action of $\mathfrak{A}$ on $\mathbb{R}^{\Omega}$ is defined by $Z(\mathfrak{f}(F))=\mathfrak{f}(Z(F))$ for all $\mathfrak{f} \in \mathfrak{E}$ and $F \in \mathbb{R}^{\Omega}$, and linear extension. More explicitly, for $\mathfrak{f} \in \mathfrak{E}, F \in \mathbb{R}^{\Omega}$, and $S \in \Omega$,

$$
(\mathfrak{f}(F))(S)=F\left(\mathfrak{f}^{-1}(S)\right)=\sum\left\{F\left(S^{\prime}\right): S^{\prime} \in \Omega \text { and } \mathfrak{f}\left(S^{\prime}\right)=S\right\} .
$$

Consider an element of $\mathfrak{A}$ of the form $\mathcal{L}=\sum_{i=1}^{N} \lambda_{i}\left(\mathfrak{f}_{i}-1\right)$ with all $\lambda_{i}>0$. Let $\lambda_{i} \leq L$ for all $i \in[N]$, and let $K=\sum_{i=1}^{N} \lambda_{i}$. The power series

$$
\exp (t \mathcal{L})=\mathrm{e}^{-K t} \sum_{n=0}^{\infty} \frac{t^{n}}{n !}\left[\sum_{i=1}^{N} \lambda_{i} \mathfrak{f}_{i}\right]^{n}=\sum_{\mathfrak{f} \in \mathfrak{E}} P_{\mathfrak{f}}(t) \cdot \mathfrak{f}
$$

in $\mathfrak{A}[[t]]$ is such that for each $\mathfrak{f} \in \mathfrak{E}, P_{\mathfrak{f}}(t) \in \mathbb{R}[[t]]$ is dominated coefficientwise by $\exp ((L N-K) t)$. Thus $\exp (t \mathcal{L}) \in \mathfrak{A}[[t]]$ converges for all $t \geq 0$. The semigroup of transformations generated by $\mathcal{L}$ is $\exp (t \mathcal{L})$.

To check claim (3), we will show that the semigroup $T(t)$ of the SEP preserves stability for all $t \geq 0$; that is, if $Z\left(\varphi_{0}\right)$ is stable, then $Z\left(\varphi_{t}\right)=T(t) Z\left(\varphi_{0}\right)$ is stable for all $t \geq 0$. This reduces to the case of a single pair $\{i, j\} \in E$, as follows. If $\mathcal{M}_{1}$ and $\mathcal{M}_{2}$ are Markov chains on the same finite state space with semigroups $T_{1}(t)$ and $T_{2}(t)$ generated by $\mathcal{L}_{1}$ and $\mathcal{L}_{2}$, then the semigroup generated by $\mathcal{L}_{1}+\mathcal{L}_{2}$ is

$$
T(t)=\lim _{n \rightarrow \infty}\left[T_{1}(t / n) T_{2}(t / n)\right]^{n},
$$

by the Trotter product formula. By Hurwitz's Theorem, it follows that if $T_{i}(t)$ preserves stability for all $t \geq 0$ and $i \in\{1,2\}$, then $T(t)$ preserves stability for all $t \geq 0$. By repeated application of this argument, in order to show that the SEP semigroup $T(t)=\exp (t \mathcal{L})$ preserves stability for all $t \geq 0$ it is enough to show that for each $\{i, j\} \in E, T_{i j}(t)=\exp \left(t \lambda_{i j}\left(\tau_{i j}-1\right)\right)$ preserves stability for all $t \geq 0$. Now, since $\tau_{i j}^{2}=1$,

$$
T_{i j}(t)=\left(\frac{1+\mathrm{e}^{-2 \lambda_{i j} t}}{2}\right)+\left(\frac{1-\mathrm{e}^{-2 \lambda_{i j} t}}{2}\right) \cdot \tau_{i j} .
$$


By Lemma 4.3, this preserves stability for all $t \geq 0$. This proves Theorem 7.1.

7.3. Further observations. In verifying the hypotheses of the Feder-Mihail Theorem, we used the fact that if $f \in \mathfrak{S}_{\mathbb{R}}[\mathbf{x}]^{\mathrm{MA}}$ is multiaffine and real stable, then $\Delta_{i j} f(\mathbf{a}) \geq 0$ for all $\{i, j\} \subseteq E$ and $\mathbf{a} \in \mathbb{R}^{m}$, by Theorem 3.1. In fact, we only needed the weaker hypothesis that $\Delta_{i j} f(\mathbf{a}) \geq 0$ for all $\{i, j\} \subseteq E$ and $\mathbf{a} \in \mathbb{R}^{m}$ with $\mathbf{a}>\mathbf{0}$. A multiaffine real polynomial satisfying this weaker condition is a Rayleigh polynomial. (This terminology is by analogy with the Rayleigh monotonicity property of electrical networks; see Definition 2.5 of [7] and the references cited therein. Multiaffine real stable polynomials are also called strongly Rayleigh.) The class of probability distributions $\varphi$ such that $Z(\varphi)$ is homogeneous, multiaffine, and Rayleigh meets all the conditions of the Feder-Mihail Theorem. It follows that all such distributions are NA.

Claim (2) above can be generalized in another way: the hypothesis of homogeneity can be removed, as follows. Let $\mathbf{y}=\left(y_{1}, \ldots, y_{m}\right)$, and let $e_{j}(\mathbf{y})$ be the $j$ th elementary symmetric function of the $\mathbf{y}$. Given a multiaffine polynomial $f=\sum_{S \subseteq[m]} c(S) \mathbf{x}^{S}$, the symmetric homogenization of $f$ is the polynomial $f_{\text {sh }}(\mathbf{x}, \mathbf{y}) \in \mathbb{C}[\mathbf{x}, \mathbf{y}]^{\mathrm{MA}}$ defined by

$$
f_{\mathrm{sh}}(\mathbf{x}, \mathbf{y})=\sum_{S \subseteq[m]} c(S) \mathbf{x}^{S}\left(\begin{array}{c}
m \\
|S|
\end{array}\right)^{-1} e_{m-|S|}(\mathbf{y}) .
$$

Note that $f_{\mathrm{sh}}$ is homogeneous of degree $m$, and $f_{\mathrm{sh}}(\mathbf{x}, \mathbf{1})=f(\mathbf{x})$.

Proposition 7.2 (Theorem 4.2 of $[7]$ ). If $f \in \mathfrak{S}_{\mathbb{R}}[\mathbf{x}]^{\mathrm{MA}}$ is multiaffine and real stable, then $f_{\mathrm{sh}} \in \mathfrak{S}_{\mathbb{R}}[\mathbf{x}, \mathbf{y}]^{\mathrm{MA}}$ is homogeneous, multiaffine, and real stable.

(We omit the proof.)

Corollary 7.3 (Theorem 4.9 of [7]). Let $\varphi: \Omega \rightarrow[0, \infty)$ be such that $Z(\varphi)$ is nonzero, multiaffine, and real stable. Then for all $\mathbf{a} \in \mathbb{R}^{m}$ with $\mathbf{a}>0$, $\varphi^{\mathbf{a}}$ is $N A$.

Proof. By Proposition $7.2, Z_{\mathrm{sh}}(\varphi ; \mathbf{x}, \mathbf{y})$ is nonzero, homogeneous, multiaffine, and real stable. This is the partition function for $\psi:\{0,1\}^{[2 m]} \rightarrow[0, \infty)$ given by $\psi(S)=\left(\begin{array}{c}m \\ |S \cap[m]|\end{array}\right)^{-1} \varphi(S \cap[m])$. By claim (2) above, $\psi^{\mathbf{a}}$ is NA for all $\mathbf{a} \in \mathbb{R}^{2 m}$ with $\mathbf{a}>\mathbf{0}$. By considering those $\mathbf{a} \in \mathbb{R}^{2 m}$ for which $a_{i}=1$ for all $m+1 \leq i \leq 2 m$, it follows that $\varphi^{\mathbf{a}}$ is NA for all $\mathbf{a} \in \mathbb{R}^{m}$ with $\mathbf{a}>0$.

Corollary 7.4 (Theorem 5.2 of [7]). If the initial distribution $\varphi_{0}$ of an SEP is such that $Z(\varphi)$ is stable (but not necessarily homogeneous), then $Z\left(\varphi_{t}\right)$ is stable, and hence $\varphi_{t}$ is $N A$, for all $t \geq 0$.

It is natural to try extending these results to asymmetric exclusion processes. For $(i, j) \in \Lambda^{2}$ define $\mathfrak{t}_{i j} \in \mathfrak{E}$ by $\mathfrak{t}_{i j}(S)=S \circ \tau_{i j}$ if $S(i)=1$ and $S(j)=0$, and $\mathfrak{t}_{i j}(S)=S$, otherwise, for all $S \in \Omega$. That is, $\mathfrak{t}_{i j}$ makes a particle hop from site $i$ to site $j$, if possible. Let $E$ be a set of ordered pairs in $\Lambda^{2}$, and for $(i, j) \in E$ let $\lambda_{i j}>0$. An asymmetric exclusion process is a Markov chain on $\Omega$ with semigroup $T(t)=\exp (t \mathcal{L})$ generated by something of the form

$$
\mathcal{L}=\sum_{(i, j) \in E} \lambda_{i j}\left(\mathfrak{t}_{i j}-1\right)
$$


By the argument for claim (3) above, in order to show that $T(t)$ preserves stability for all $t \geq 0$, it suffices to do so for the two-site semigroup $T_{\{1,2\}}(t)=$ $\exp \left(t \mathcal{L}_{\{1,2\}}\right)$ generated by

$$
\mathcal{L}_{\{1,2\}}=\lambda_{12}\left(\mathfrak{t}_{12}-1\right)+\lambda_{21}\left(\mathfrak{t}_{21}-1\right) .
$$

Exercise 7.5 (Strengthening Remark 5.3 of [7]). With the notation above, let $\lambda=\lambda_{12}+\lambda_{21}, \beta_{12}=\lambda_{12} / \lambda$, and $\beta_{21}=\lambda_{21} / \lambda$.

(a) In $\mathfrak{A}, \mathfrak{t}_{12}+\mathfrak{t}_{21}=1+\tau_{12}$.

(b) If $\omega$ is any word in $\left\{\mathfrak{t}_{12}, \mathfrak{t}_{21}\right\}^{n}$, then $\mathfrak{t}_{12} \omega=\mathfrak{t}_{12}$ and $\mathfrak{t}_{21} \omega=\mathfrak{t}_{21}$.

(c) The semigroup generated by $\mathcal{L}_{\{1,2\}}$ is

$$
T_{\{1,2\}}(t)=\mathrm{e}^{-\lambda t}+\left(1-\mathrm{e}^{-\lambda t}\right)\left(\beta_{12} \mathfrak{t}_{12}+\beta_{21} \mathfrak{t}_{21}\right)
$$

(d) The semigroup $T_{\{1,2\}}(t)$ preserves stability for all $t \geq 0$ if and only if $\beta_{12}=$ $\beta_{21}=1 / 2$, in which case it reduces to the SEP (of rate $\lambda / 2$ ).

Thus, even the slightest asymmetry ruins preservation of stability by the SEP!

Finally, we consider an SEP in which particle number is not conserved. For $i \in \Lambda$ define $\mathfrak{a}_{i}, \mathfrak{a}_{i}^{*} \in \mathfrak{E}$ as follows: for $S \in \Omega$ and $j \in \Lambda$, let $\left(\mathfrak{a}_{i}(S)\right)(j)=\left(\mathfrak{a}_{i}^{*}(S)\right)(j)=S(j)$ if $j \neq i$, and $\left(\mathfrak{a}_{i}(S)\right)(i)=0$ and $\left(\mathfrak{a}_{i}^{*}(S)\right)(i)=1$. That is, $\mathfrak{a}_{i}$ annihilates a particle at site $i$, and $\mathfrak{a}_{i}^{*}$ creates a particle at site $i$, if possible.

An SEP with particle creation and annihilation is a Markov chain on $\Omega$ with semigroup $T(t)=\exp (t \mathcal{L})$ generated by something of the form

$$
\mathcal{L}=\sum_{\{i, j\} \in E} \lambda_{i j}\left(\tau_{i j}-1\right)+\sum_{i \in \Lambda}\left[\theta_{i}\left(\mathfrak{a}_{i}-1\right)+\theta_{i}^{*}\left(\mathfrak{a}_{i}^{*}-1\right)\right],
$$

in which the first sum is the generator of the SEP in Theorem 7.1 and $\theta_{i}, \theta_{i}^{*} \geq 0$ for each $i \in \Lambda$.

By the argument for claim (3) above, to show that this $T(t)$ preserves stability for all $t \geq 0$, it suffices to do so for the one-site semigroups generated by $\mathcal{L}_{1}=\theta\left(\mathfrak{a}_{1}-1\right)$ and $\mathcal{L}_{1}^{*}=\theta\left(\mathfrak{a}_{1}^{*}-1\right)$, respectively.

Exercise 7.6. The semigroups generated by $\mathcal{L}_{1}$ and $\mathcal{L}_{1}^{*}$ are

$$
T_{1}(t)=\mathrm{e}^{-\theta t}+\left(1-\mathrm{e}^{-\theta t}\right) \mathfrak{a}_{1} \text { and } T_{1}^{*}(t)=\mathrm{e}^{-\theta t}+\left(1-\mathrm{e}^{-\theta t}\right) \mathfrak{a}_{1}^{*},
$$

respectively. Both $T_{1}(t)$ and $T_{1}^{*}(t)$ preserve stability.

Corollary 7.7. If the initial distribution $\varphi_{0}$ of an SEP with particle creation and annihilation is such that $Z(\varphi)$ is stable, then $Z\left(\varphi_{t}\right)$ is stable, and hence $\varphi_{t}$ is $N A$, for all $t \geq 0$.

\section{INEQUALITIES FOR MIXED DISCRIMINANTS}

This section summarizes a powerful application of stable polynomials from a 2008 paper of Gurvits [12].

We will use without mention the facts that $\log$ and exp are strictly increasing functions on $(0, \infty)$. A function $\rho: I \rightarrow \mathbb{R}$ defined on an interval $I \subseteq \mathbb{R}$ is convex provided that for all $a_{1}, a_{2} \in I, \rho\left(\left(a_{1}+a_{2}\right) / 2\right) \leq\left(\rho\left(a_{1}\right)+\rho\left(a_{2}\right)\right) / 2$. It is strictly convex if it is convex and equality holds here only when $a_{1}=a_{2}$. A function $\rho: I \rightarrow \mathbb{R}$ is (strictly) concave if $-\rho$ is (strictly) convex. For example, for positive reals $a_{1}, a_{2}>0$ one has $\left(\sqrt{a_{1}}-\sqrt{a_{2}}\right)^{2} \geq 0$, with equality only if $a_{1}=a_{2}$. It follows that $\log \left(\left(a_{1}+a_{2}\right) / 2\right) \geq\left(\log \left(a_{1}\right)+\log \left(a_{2}\right)\right) / 2$, with equality only if $a_{1}=a_{2}$. That is, $\log$ is strictly concave. 
Jensen's Inequality (Theorem 90 of [14]). Let $\rho: I \rightarrow \mathbb{R}$ be defined on an interval $I \subseteq \mathbb{R}$, let $a_{i} \in I$ for $i \in[n]$, and let $b_{i}>0$ for $i \in[n]$ be such that $\sum_{i=1}^{n} b_{i}=1$. If $\rho$ is convex, then

$$
\rho\left(\sum_{i=1}^{n} b_{i} a_{i}\right) \leq \sum_{i=1}^{n} b_{i} \rho\left(a_{i}\right)
$$

If $\rho$ is strictly convex and equality holds, then $a_{1}=a_{2}=\cdots=a_{n}$.

For integer $d \geq 1$, let $\mathrm{G}(d)=(1-1 / d)^{d-1}$, and let $\mathrm{G}(0)=1$. Note that $\mathrm{G}(1)=0^{0}=1$, and that $\mathrm{G}(d)$ is a strictly decreasing function for $d \geq 1$. For homogeneous $f \in \mathbb{R}[\mathbf{x}]$ with nonnegative coefficients, define the capacity of $f$ to be

$$
\operatorname{cap}(f)=\inf _{\mathbf{c}>\mathbf{0}} \frac{f(\mathbf{c})}{c_{1} \cdots c_{m}},
$$

with the infimum over the set of all $\mathbf{c} \in \mathbb{R}^{m}$ with $c_{i}>0$ for all $i \in[m]$.

Lemma 8.1 (Lemma 3.2 of [12]). Let $f=\sum_{i=0}^{d} b_{i} x \in \mathbb{R}[x]$ be a nonzero univariate polynomial of degree $d$ with nonnegative coefficients. If $f$ is real stable, then $b_{1}=$ $f^{\prime}(0) \geq \mathrm{G}(d) \operatorname{cap}(f)$, and if $\operatorname{cap}(f)>0$, then equality holds if and only if $d \leq 1$ or $f(x)=b_{d}(x+\xi)^{d}$ for some $\xi>0$.

Proof. If $\operatorname{cap}(f)=0$, then there is nothing to prove, so assume that $\operatorname{cap}(f)>0$. If $d=0$, then $f^{\prime}(0)=b_{1}=0=\mathrm{G}(0) \operatorname{cap}(f)$, and if $d=1$, then $f^{\prime}(0)=b_{1}=$ $\mathrm{G}(1) \operatorname{cap}(f)$, so assume that $d \geq 2$. If $f(0)=0$, then $f^{\prime}(0)=\lim _{c \rightarrow 0} f(c) / c \geq$ $\operatorname{cap}(f)>\mathrm{G}(d) \operatorname{cap}(f)$. Thus, assume that $d \geq 2$ and $f(0)=b_{0}>0$. We may rescale the polynomial so that $b_{0}=1$. Now there are $a_{i}>0$ for $i \in[d]$ such that

$$
f(x)=\prod_{i=1}^{d}\left(1+a_{i} x\right),
$$

and $b_{1}=a_{1}+\cdots+a_{d}$. For any $c>0$ we have

$$
\frac{\log (\operatorname{cap}(f) c)}{d} \leq \frac{\log (f(c))}{d}=\frac{1}{d} \sum_{i=1}^{d} \log \left(1+a_{i} c\right) \leq \log \left(1+\frac{b_{1} c}{d}\right),
$$

by Jensen's Inequality. It follows that $\operatorname{cap}(f) c \leq\left(1+b_{1} c / d\right)^{d}$ for all $c>0$. Let $g(x)=\left(1+b_{1} x / d\right)^{d}$. Elementary calculus shows that

$$
\operatorname{cap}(g)=\inf _{c>0} \frac{g(c)}{c}=\frac{g\left(c_{*}\right)}{c_{*}}=\frac{b_{1}}{\mathrm{G}(d)}, \text { in which } c_{*}=\frac{d}{b_{1}(d-1)} .
$$

Since $\operatorname{cap}(f) \leq \operatorname{cap}(g)$, this yields the stated inequality. If equality holds, then equality holds in the application of Jensen's Inequality, and so $f$ has the stated form.

Lemma 8.2 (Theorem 4.10 of [12]). Let $f \in \mathfrak{S}_{\mathbb{R}}\left[x_{1}, \ldots, x_{m}\right]$ be real stable, with nonnegative coefficients, and homogeneous of degree $k$. Let $g=\left.\partial_{m} f\right|_{x_{m}=0}$. Then

$$
\operatorname{cap}(g) \geq \mathrm{G}\left(\operatorname{deg}_{m}(f)\right) \operatorname{cap}(f) .
$$

Proof. We may assume that $d=\operatorname{deg}_{m}(f) \geq 1$. Let $c_{i}>0$ for $i \in[m-1]$, and let $p_{\mathbf{c}}(x)=f\left(c_{1}, \ldots, c_{m-1}, x\right)$. Since $f$ has nonnegative coefficients, $p_{\mathbf{c}}$ has degree $d \geq 1$. By specialization, $p_{\mathbf{c}}$ is real stable. Lemma 8.1 implies that

$$
g(\mathbf{c})=p_{\mathbf{c}}^{\prime}(0) \geq \mathrm{G}(d) \operatorname{cap}\left(p_{\mathbf{c}}\right) \geq \mathrm{G}(d) \operatorname{cap}(f)
$$


for all $\mathbf{c} \in \mathbb{R}^{m-1}$ with $\mathbf{c}>\mathbf{0}$. If $m=1$ or $k=1$, then $g=\operatorname{cap}(g)$ is a constant. If $m \geq 2$ and $k \geq 2$, then for any such $\mathbf{c}$ let $b=\left(c_{1} \cdots c_{m-1}\right)^{-1 /(k-1)}$. Since $g$ is homogeneous of degree $k-1$,

$$
\frac{g(\mathbf{c})}{c_{1} \cdots c_{m-1}}=g\left(b c_{1}, \ldots, b c_{m-1}\right) \geq \mathrm{G}(d) \operatorname{cap}(f) .
$$

It follows that $\operatorname{cap}(g) \geq \mathrm{G}(d) \operatorname{cap}(f)$.

Theorem 8.3 (Theorem 2.4 of [12]). Let $f \in \mathfrak{S}_{\mathbb{R}}\left[x_{1}, \ldots, x_{m}\right]$ be real stable, with nonnegative coefficients, and homogeneous of degree $m$. Let $\operatorname{deg}_{i}(f)=d_{i}$ and $e_{i}=\min \left\{i, d_{i}\right\}$ for each $i \in[m]$. Then

$$
\boldsymbol{\partial}^{\mathbf{1}} f(\mathbf{0}) \geq \operatorname{cap}(f) \prod_{i=2}^{m} \mathrm{G}\left(e_{i}\right) .
$$

Proof. Let $g_{m}=f$ and let $g_{i-1}=\left.\partial_{i} g_{i}\right|_{x_{i}=0}$ for all $i \in[m]$. By contraction and specialization, $g_{i}$ is real stable for each $i \in[m]$. Notice that $g_{0}=\boldsymbol{\partial}^{\mathbf{1}} f(\mathbf{0})=\operatorname{cap}\left(g_{0}\right)$. By Lemma 8.2. $\operatorname{cap}\left(g_{i-1}\right) \geq \operatorname{cap}\left(g_{i}\right) \cdot \mathrm{G}\left(\operatorname{deg}_{i}\left(g_{i}\right)\right)$ for each $i \in[m]$. But $\operatorname{deg}_{i}\left(g_{i}\right) \leq$ $\operatorname{deg}_{i}(f)=d_{i}$, and $\operatorname{deg}_{i}\left(g_{i}\right)$ is at most the total degree of $g_{i}$, which is $i$. Hence $\operatorname{deg}_{i}\left(g_{i}\right) \leq e_{i}$, and thus $\mathrm{G}\left(\operatorname{deg}_{i}\left(g_{i}\right)\right) \geq \mathrm{G}\left(e_{i}\right)$. Thus $\operatorname{cap}\left(g_{i-1}\right) \geq \operatorname{cap}\left(g_{i}\right) \cdot \mathrm{G}\left(e_{i}\right)$ for each $i \in[m]$. Combining these inequalities (and $\mathrm{G}\left(e_{1}\right)=1$ ) gives the result.

With the notation of Theorem 8.3, since $e_{i} \leq i$ for all $i \in[m]$ and $\mathrm{G}(d)$ is a decreasing function of $d$, one has the inequality

$$
\prod_{i=2}^{m} \mathrm{G}\left(e_{i}\right) \geq \prod_{i=2}^{m} \mathrm{G}(i)=\prod_{i=2}^{m}\left(\frac{i-1}{i}\right)^{i-1}=\frac{m !}{m^{m}} .
$$

Thus, the following corollary is immediate.

Corollary 8.4. Let $f \in \mathfrak{S}_{\mathbb{R}}\left[x_{1}, \ldots, x_{m}\right]$ be real stable, with nonnegative coefficients, and homogeneous of degree $m$. Then

$$
\partial^{\mathbf{1}} f(\mathbf{0}) \geq \frac{m !}{m^{m}} \cdot \operatorname{cap}(f) .
$$

Theorem 8.5 (Theorem 5.7 of [12]). Let $f \in \mathfrak{S}_{\mathbb{R}}\left[x_{1}, \ldots, x_{m}\right]$ be real stable, with nonnegative coefficients, and homogeneous of degree $m$. Equality holds in the bound of Corollary 8.4 if and only if there are nonnegative reals $a_{i} \geq 0$ for $i \in[\mathrm{m}]$ such that

$$
f(\mathbf{x})=\left(a_{1} x_{1}+\cdots+a_{m} x_{m}\right)^{m} .
$$

(We omit the proof, which consists of following the case of equality in Jensen's Inequality through the proof of Theorem 8.3.)

Lemma 8.6 (Fact 2.2 of [12]). Let $f \in \mathbb{R}\left[x_{1}, \ldots, x_{m}\right]$ be homogeneous of degree $m$, with nonnegative coefficients. Assume that $\partial_{i} f(\mathbf{1})=1$ for all $i \in[m]$. Then $\operatorname{cap}(f)=1$.

Proof. Let $f=\sum_{\alpha} b(\alpha) \mathbf{x}^{\alpha}$, so that if $b(\alpha) \neq 0$, then $|\alpha|=\sum_{i=1}^{m} \alpha(i)=m$. By hypothesis, for all $i \in[m], \sum_{\alpha} b(\alpha) \alpha(i)=1$. Averaging these over all $i \in[m]$ yields 
$f(\mathbf{1})=\sum_{\alpha} b(\alpha)=1$, so that $\operatorname{cap}(f) \leq 1$. Conversely, let $\mathbf{c} \in \mathbb{R}^{m}$ with $\mathbf{c}>\mathbf{0}$. Jensen's Inequality implies that

$$
\begin{aligned}
\log (f(\mathbf{c})) & =\log \left(\sum_{\alpha} b(\alpha) \mathbf{c}^{\alpha}\right) \\
& \geq \sum_{\alpha} b(\alpha) \log \left(\mathbf{c}^{\alpha}\right)=\sum_{i=1}^{m} \log \left(c_{i}\right) \sum_{\alpha} b(\alpha) \alpha(i)=\log \left(c_{1} \cdots c_{m}\right) .
\end{aligned}
$$

It follows that $\operatorname{cap}(f) \geq 1$.

Example 8.7 (van der Waerden Conjecture). An $m$-by- $m$ matrix $A=\left(a_{i j}\right)$ is doubly stochastic if all entries are nonnegative reals and every row and column sums to one. In 1926, van der Waerden conjectured that if $A$ is an $m$-by- $m$ doubly stochastic matrix then $\operatorname{per}(A) \geq m ! / m^{m}$, with equality if and only if $A=(1 / m) J$, the $m$-by- $m$ matrix in which every entry is $1 / m$. In 1981 this lower bound was proved by Falikman, and the characterization of equality was proved by Egorychev. These results follow immediately from Corollary 8.4 and Theorem 8.5, as follows. It suffices to prove the result for an $m$-by- $m$ doubly stochastic matrix $A=\left(a_{i j}\right)$ with no zero entries, by a routine limit argument. The polynomial

$$
f_{A}(\mathbf{x})=\prod_{j=1}^{m}\left(a_{1 j} x_{1}+\cdots+a_{m j} x_{m}\right)
$$

is clearly homogeneous and real stable, with nonnegative coefficients and of degree $m$, and such that $\operatorname{deg}_{i}\left(f_{A}\right)=m$ for all $i \in[m]$. Since $A$ is doubly stochastic, Lemma 8.6 implies that $\operatorname{cap}\left(f_{A}\right)=1$. Since

$$
\operatorname{per}(A)=\boldsymbol{\partial}^{\mathbf{1}} f_{A}(\mathbf{0})
$$

Corollary 8.4 and Theorem 8.5 imply the results of Falikman and Egorychev, respectively. Gurvits [12 also uses a similar argument to prove a refinement of the van der Waerden Conjecture due to Schrijver and Valiant; see also [15]. is

Given $n$-by- $n$ matrices $A_{1}, \ldots, A_{m}$, the mixed discriminant of $\mathcal{A}=\left(A_{1}, \ldots, A_{m}\right)$

$$
\operatorname{Disc}(\mathcal{A})=\left.\boldsymbol{\partial}^{\mathbf{1}} \operatorname{det}\left(x_{1} A_{1}+\cdots+x_{m} A_{m}\right)\right|_{\mathbf{x}=\mathbf{0}} .
$$

This generalizes the permanent of an $m$-by- $m$ matrix $B=\left(b_{i j}\right)$ by considering the collection of matrices $\mathcal{A}(B)=\left(A_{1}, \ldots, A_{m}\right)$ defined by $A_{h}=\operatorname{diag}\left(a_{h 1}, \ldots, a_{h m}\right)$ for each $h \in[m]$. In this case one sees that

$$
\operatorname{det}\left(x_{1} A_{1}+\cdots+x_{m} A_{m}\right)=f_{B}(\mathbf{x})
$$

with the notation of Example 8.7, and it follows that $\operatorname{Disc}(\mathcal{A}(B))=\operatorname{per}(B)$.

Example 8.8 (Bapat's Conjecture). Generalizing the van der Waerden Conjecture, in 1989 Bapat considered the set $\Omega(m)$ of $m$-tuples of $m$-by- $m$ matrices $\mathcal{A}=\left(A_{1}, \ldots, A_{m}\right)$ such that each $A_{i}$ is positive semidefinite with $\operatorname{trace} \operatorname{tr}\left(A_{i}\right)=1$, and $\sum_{i=1}^{m} A_{i}=I$. For any doubly stochastic matrix $B, \mathcal{A}(B)$ is in this set. The natural conjecture is that for all $\mathcal{A} \in \Omega(m), \operatorname{Disc}(\mathcal{A}) \geq m ! / m^{m}$, and equality is attained if and only if $\mathcal{A}=\mathcal{A}((1 / m) J)$. This was proved by Gurvits in 2006; again, it follows directly from Corollary 8.4 and Theorem 8.5. It suffices to prove the result 
for $\mathcal{A} \in \Omega(m)$ such that each $A_{i}$ is positive definite, by a routine limit argument. By Proposition 2.1, for $\mathcal{A} \in \Omega(m)$, the polynomial

$$
f_{\mathcal{A}}(\mathbf{x})=\operatorname{det}\left(x_{1} A_{1}+\cdots+x_{m} A_{m}\right)
$$

is real stable. Since each $A_{i}$ is positive definite, all coefficients of $f_{\mathcal{A}}$ are nonnegative, $f_{\mathcal{A}}$ is homogeneous of degree $m$, and $\operatorname{deg}_{i}\left(f_{A}\right)=m$ for all $i \in[m]$. Since $\mathcal{A} \in \Omega(m)$, Lemma 8.6 implies that $\operatorname{cap}\left(f_{\mathcal{A}}\right)=1$. Thus, $f_{\mathcal{A}}$ satisfies the hypothesis of Theorems 8.3 and 8.5 , and since $\operatorname{Disc}(\mathcal{A})=\boldsymbol{\partial}^{\mathbf{1}} f_{\mathcal{A}}(\mathbf{0})$, the result follows.

\section{FURTHER DIRECTIONS}

9.1. Other circular regions. Let $\Omega \subseteq \mathbb{C}^{m}$. A polynomial $f \in \mathbb{C}[\mathbf{x}]$ is $\Omega$-stable if either $f \equiv 0$ identically, or $f(\mathbf{z}) \neq 0$ for all $\mathbf{z} \in \Omega$. At this level of generality little can be said. If $\Omega=\mathcal{A}_{1} \times \cdots \times \mathcal{A}_{m}$ is a product of open circular regions, then there are Möbius transformations $z \mapsto \phi_{i}(z)=\left(a_{i} z+b_{i}\right) /\left(c_{i} z+d_{i}\right)$ such that $\phi_{i}(\mathcal{H})=\mathcal{A}_{i}$ for all $i \in[m]$. The argument in Section 4.1 shows that $f \in \mathbb{C}[\mathbf{x}]$ is $\Omega$-stable if and only if

$$
\widetilde{f}=(\mathbf{c z}+\mathbf{d})^{\operatorname{deg}(f)} \cdot f\left(\phi_{1}\left(z_{1}\right), \ldots, \phi_{m}\left(z_{m}\right)\right)
$$

is stable. In this way results about stable polynomials can be translated into results about $\Omega$-stable polynomials for any $\Omega$ that is a product of open circular regions.

Theorem 6.3 of $[5]$ is the $\Omega$-stability analogue of Theorem 5.2. We mention only two consequences of this. Let $\mathcal{D}=\{z \in \mathbb{C}:|z|<1\}$ be the open unit disc, and for $\theta \in \mathbb{R}$, let $\mathcal{H}_{\theta}=\left\{\mathrm{e}^{-\mathrm{i} \theta} z: z \in \mathcal{H}\right\}$. Thus $\mathcal{H}_{0}=\mathcal{H}$, and $\mathcal{H}_{\pi / 2}$ is the open right half-plane. A $\mathcal{D}^{m}$-stable polynomial is called Schur stable, and a $\mathcal{H}_{\pi / 2}^{m}$-stable polynomial is called Hurwitz stable.

Proposition 9.1 (Remark 6.1 of $[5]$ ). Fix $\kappa \in \mathbb{N}^{m}$, and let $T: \mathbb{C}[\mathbf{x}] \leq \kappa \rightarrow \mathbb{C}[\mathbf{x}]$ be a linear transformation. The following are equivalent:

(a) $T$ preserves Schur stability.

(b) $T\left((\mathbf{1}+\mathbf{x y})^{\kappa}\right)$ is Schur stable in $\mathbb{C}[\mathbf{x}, \mathbf{y}]$.

Proposition 9.2 (Remark 6.1 of $[5]$.). Fix $\kappa \in \mathbb{N}^{m}$, and let $T: \mathbb{C}[\mathbf{x}]^{\leq \kappa} \rightarrow \mathbb{C}[\mathbf{x}]$ be a linear transformation. The following are equivalent:

(a) $T$ preserves Hurwitz stability.

(b) $T\left((\mathbf{1}+\mathbf{x y})^{\kappa}\right)$ is Hurwitz stable in $\mathbb{C}[\mathbf{x}, \mathbf{y}]$.

9.2. Applications of Theorem 5.4. It is natural to consider a multivariate analogue of the multiplier sequences studied by Pólya and Schur. Let $\lambda: \mathbb{N}^{m} \rightarrow \mathbb{R}$, and define a linear transformation $T_{\lambda}: \mathbb{C}[\mathbf{x}] \rightarrow \mathbb{C}[\mathbf{x}]$ by $T_{\lambda}\left(\mathbf{x}^{\alpha}\right)=\lambda(\alpha) \mathbf{x}^{\alpha}$ for all $\alpha \in \mathbb{N}^{m}$, and linear extension. For which $\lambda$ does $T_{\lambda}$ preserve real stability? The answer: just the ones you get from the Pólya-Schur Theorem, and no more.

Theorem 9.3 (Theorem 1.8 of [4]). Let $\lambda: \mathbb{N}^{m} \rightarrow \mathbb{R}$. Then $T_{\lambda}$ preserves real stability if and only if there are univariate multiplier sequences $\lambda_{i}: \mathbb{N} \rightarrow \mathbb{R}$ for $i \in[m]$ and $\epsilon \in\{-1,+1\}$ such that

$$
\lambda(\alpha)=\lambda_{1}(\alpha(1)) \cdots \lambda_{m}(\alpha(m))
$$

for all $\alpha \in \mathbb{N}^{m}$, and either $\epsilon^{|\alpha|} \lambda(\alpha) \geq 0$ for all $\alpha \in \mathbb{N}^{m}$, or $\epsilon^{|\alpha|} \lambda(\alpha) \leq 0$ for all $\alpha \in \mathbb{N}^{m}$. 
Theorem 5.4 (and similarly Propositions 9.1 and 9.2) can be used to derive a wide variety of results of the form, such-and-such an operation preserves stability (or Schur or Hurwitz stability). Here is a short account of Hinkkanen's proof of the Lee-Yang Circle Theorem, taken from Section 8 of $[6]$.

For $f, g \in \mathbb{C}[\mathbf{x}]^{\mathrm{MA}}$, say $f=\sum_{S \subseteq[m]} a(S) \mathbf{x}^{S}$ and $g=\sum_{S \subseteq[m]} b(S) \mathbf{x}^{S}$, let

$$
f \bullet g=\sum_{S \subseteq[m]} a(S) b(S) \mathbf{x}^{S}
$$

be the Schur-Hadamard product of $f$ and $g$.

Theorem 9.4 (Hinkkanen, Theorem 8.5 of $[6]$ ). If $f, g \in \mathbb{C}[\mathbf{x}]^{\mathrm{MA}}$ are Schur stable, then $f \bullet g$ is Schur stable.

Proof. Let $T_{g}: \mathbb{C}[\mathbf{x}]^{\mathrm{MA}} \rightarrow \mathbb{C}[\mathbf{x}]^{\mathrm{MA}}$ be defined by $f \mapsto f \bullet g$. By Proposition 9.1 , to show that $T_{g}$ preserves Schur stability, it suffices to show that $T_{g}\left((\mathbf{1}+\mathbf{x y})^{[m]}\right)$ is Schur stable. Clearly, $T_{g}\left((\mathbf{1}+\mathbf{x y})^{[m]}\right)=g\left(x_{1} y_{1}, \ldots, x_{m} y_{m}\right)$ is Schur stable since $g(\mathbf{x})$ is. Hence $T_{g}$ preserves Schur stability, and so $f \bullet g$ is Schur stable.

Theorem 9.5 (Lee-Yang Circle Theorem, Theorem 8.4 of [6]). Let $A=\left(a_{i j}\right)$ be a Hermitian m-by-m matrix with $\left|a_{i j}\right| \leq 1$ for all $i, j \in[m]$. Then the polynomial

$$
f(\mathbf{x})=\sum_{S \subseteq[m]} \mathbf{x}^{S} \prod_{i \in S} \prod_{j \notin S} a_{i j}
$$

is Schur stable. The diagonalization $g(x)=f(x, \ldots, x)$ is such that $x^{m} g(1 / x)=$ $g(x)$, and it follows that all roots of $g(x)$ are on the unit circle.

Proof. For $i<j$ in $[m]$, let

$$
f_{i j}=\left(1+a_{i j} x_{i}+\overline{a_{i j}} x_{j}+x_{i} x_{j}\right) \prod_{h \in[m] \backslash\{i, j\}}\left(1+x_{h}\right) .
$$

One can check that each $f_{i j}$ is Schur stable. The polynomial $f(\mathbf{x})$ is the SchurHadamard product of all the $f_{i j}$ for $i<j$ in $[m]$. By Theorem 9.4, $f(\mathbf{x})$ is Schur stable.

Section 8 of [6] contains many many more results of this nature.

9.3. A converse to the Grace-Walsh-Szegő Theorem. The argument of Sections 4.2 and 4.3 can be used to prove the following.

Exercise 9.6. If $f \in \mathfrak{S}[\mathbf{x}]^{\mathrm{MA}}$ is multiaffine and stable, then

$$
T_{\mathcal{S}(m)}(f)=\frac{1}{m !} \sum_{\sigma \in \mathcal{S}(m)} \sigma(f)
$$

is multiaffine and stable.

This is in fact equivalent to the Grace-Walsh-Szegö Theorem, since for all $f \in$ $\mathbb{C}[\mathbf{x}]^{\mathrm{MA}}, T_{\mathcal{S}(m)} f(\mathbf{x})=\operatorname{Pol}_{m} f(x, \ldots, x)$. For which transitive permutation groups $G \leq \mathcal{S}(m)$ does the linear transformation $T_{G}=|G|^{-1} \sum_{\sigma \in G} \sigma$ preserve stability? The answer: not many, and they give nothing new.

Theorem 9.7 (Theorem 6 of 9 ). Let $G \leq \mathcal{S}(m)$ be a transitive permutation group such that $T_{G}$ preserves stability. Then $T_{G}=T_{\mathcal{S}(m)}$. 
9.4. Phase and support theorems. A polynomial $f \in \mathbb{C}[\mathbf{x}]$ has definite parity if every monomial $\mathbf{x}^{\alpha}$ occurring in $f$ has total degree of the same parity - all are even, or all are odd.

Theorem 9.8 (Theorem 6.2 of $[10]$ ). Let $f \in \mathbb{C}[\mathbf{x}]$ be Hurwitz stable and with definite parity. Then there is a phase $0 \leq \theta<2 \pi$ such that $\mathrm{e}^{-\mathrm{i} \theta} f(\mathbf{x})$ has only real nonnegative coefficients.

The support of $f=\sum_{\alpha} c(\alpha) \mathbf{x}^{\alpha}$ is $\operatorname{supp}(f)=\left\{\alpha \in \mathbb{N}^{m}: c(\alpha) \neq 0\right\}$. Let $\delta_{i}$ denote the unit vector with a one in the $i$ th coordinate, and for $\alpha \in \mathbb{Z}^{n}$, let $|\alpha|=\sum_{i=1}^{m}|\alpha(i)|$. A jump system is a subset $\mathcal{J} \subseteq \mathbb{Z}^{m}$ satisfying the following two-step axiom:

(J) If $\alpha, \beta \in \mathcal{J}$ and $i \in[m]$ and $\epsilon \in\{-1,+1\}$ are such that $\alpha^{\prime}=\alpha+\epsilon \delta_{i}$ satisfies $\left|\alpha^{\prime}-\beta\right|<|\alpha-\beta|$, then either $\alpha^{\prime} \in \mathcal{J}$ or there exists $j \in[m]$ and $\varepsilon \in\{-1,+1\}$ such that $\alpha^{\prime \prime}=\alpha^{\prime}+\varepsilon \delta_{j} \in \mathcal{J}$ and $\left|\alpha^{\prime \prime}-\beta\right|<\left|\alpha^{\prime}-\beta\right|$.

Jump systems generalize some more familiar combinatorial objects. A jump system contained in $\{0,1\}^{m}$ is a delta-matroid. A delta-matroid $\mathcal{J}$ for which $|\alpha|$ is constant for all $\alpha \in \mathcal{J}$ is the set of bases of a matroid. For bases of matroids, the two-step axiom $(\mathrm{J})$ reduces to the basis exchange axiom familiar from linear algebra: if $A, B \in \mathcal{J}$ and $a \in A \backslash B$, then there exists $b \in B \backslash A$ such that $(A \backslash\{a\}) \cup\{b\}$ is in $\mathcal{J}$.

Theorem 9.9 (Theorem 3.2 of $[8]$ ). If $f \in \mathfrak{S}[\mathbf{x}]$ is stable, then the support $\operatorname{supp}(f)$ is a jump system.

Recall from Section 7 that for multiaffine polynomials with nonnegative coefficients, real stability implies the Rayleigh property. A set system $\mathcal{J}$ is convex when $A, B \in \mathcal{J}$ and $A \subseteq B$ imply that $C \in \mathcal{J}$ for all $A \subseteq C \subseteq B$.

Theorem 9.10 (Section 4 of [18]). Let $f=\sum_{S \subseteq[m]} c(S) \mathbf{x}^{S}$ be multiaffine with real nonnegative coefficients, and assume that $f$ is Rayleigh.

(a) The support $\operatorname{supp}(f)$ is a convex delta-matroid.

(b) The coefficients are log-submodular: for all $A, B \subseteq[m]$,

$$
c(A \cap B) c(A \cup B) \leq c(A) c(B) .
$$

\section{ABOut THE AUTHOR}

David Wagner received a B.Sc. from Simon Fraser University in 1985 and a Ph.D. from the Massachusetts Institute of Technology in 1990. He has been at the University of Waterloo ever since. In 2008, together with Peter Cameron, Bill Jackson, Alex Scott, and Alan Sokal, he co-organized a six month program on Combinatorics and Statistical Mechanics at the Isaac Newton Institute.

\section{REFERENCES}

1. J. Borcea and P. Brändén, Applications of stable polynomials to mixed determinants: Johnson's conjectures, unimodality, and symmetrized Fischer products, Duke Math. J. 143 (2008), 205-223. MR2420507 (2009b:15015)

2. J. Borcea and P. Brändén, Lee-Yang problems and the geometry of multivariate polynomials, Lett. Math. Phys. 86 (2008), 53-61. MR2460727 (2010e:82038)

3. J. Borcea and P. Brändén, Pólya-Schur master theorems for circular domains and their boundaries, Ann. of Math. 170 (2009), 465-492. MR2521123 (2010g:30004)

4. J. Borcea and P. Brändén, Multivariate Pólya-Schur classification problems in the Weyl algebra, Proc. London Math. Soc. 101 (2010), 73-104. 
5. J. Borcea and P. Brändén, The Lee-Yang and Pólya-Schur programs I: Linear operators preserving stability, Invent. Math. 177 (2009), 541-569. MR2534100

6. J. Borcea and P. Brändén, The Lee-Yang and Pólya-Schur programs II: Theory of stable polynomials and applications Comm. Pure Appl. Math. 62 (2009), 1595-1631. MR2569072

7. J. Borcea, P. Brändén, and T.M. Liggett, Negative dependence and the geometry of polynomials, J. Amer. Math. Soc. 22 (2009), 521-567. MR2476782 (2010b:62215)

8. P. Brändén, Polynomials with the half-plane property and matroid theory, Adv. Math. 216 (2007), 302-320. MR2353258 (2008h:05022)

9. P. Brändén and D.G. Wagner, A converse to the Grace-Walsh-Szegö theorem, Math. Proc. Camb. Phil. Soc. 147 (2009), 447-453. MR2525937

10. Y.-B. Choe, J.G. Oxley, A.D. Sokal, and D.G. Wagner, Homogeneous polynomials with the half-plane property, Adv. in Appl. Math. 32 (2004), 88-187. MR2037144 (2005d:05043)

11. L. Gårding, An inequality for hyperbolic polynomials, J. Math. Mech. 8 (1959), 957-965. MR 0113978 (22:4809)

12. L. Gurvits, Van der Waerden/Schrijver-Valiant like conjectures and stable (aka hyperbolic) homogeneous polynomials: one theorem for all. With a corrigendum, Electron. J. Combin. 15 (2008), R66 (26 pp). MR2411443(2009e:15015)

13. F.R. Harvey and H.B. Lawson Jr., Hyperbolic polynomials and the Dirichlet problem, http://arxiv.org/abs/0912.5220.

14. G. Hardy, J.E. Littlewood, and G. Pólya, "Inequalities (Second Edition)," Cambridge University Press, Cambridge, 1952. MR0046395(13:727e)

15. M. Laurent and A. Schrijver, On Leonid Gurvits' proof for permanents, http://homepages.cwi.nl/ lex/files/perma5.pdf

16. Q.I. Rahman and G. Schmeisser, "Analytic Theory of Polynomials," London Math. Soc. Monographs (N.S.) 26, Oxford University Press, New York, 2002. MR.1954841 (2004b:30015)

17. V. Scheidemann, "Introduction to Complex Analysis in Several Variables," Birkhäuser, Basel, 2005. MR2176976 (2006i:32001)

18. D.G. Wagner, Negatively correlated random variables and Mason's conjecture for independent sets in matroids, Ann. of Combin. 12 (2008), 211-239. MR.2428906 (2009f:05053)

19. D.G. Wagner and Y. Wei, A criterion for the half-plane property, Discrete Math. 309 (2009), 1385-1390. MR2510546 (2010h:05076)

Department of Combinatorics and Optimization, University of Waterloo, Waterloo, Ontario, Canada N2L 3G1

E-mail address: dgwagner@math.uwaterloo.ca 